\title{
Generalizing empirical adequacy II: partial structures
}

\author{
Sebastian Lutz ${ }^{1}$ (D) \\ Received: 17 May 2018 / Accepted: 31 January 2019 / Published online: 15 February 2019 \\ (c) The Author(s) 2019
}

\begin{abstract}
I show that extant attempts to capture and generalize empirical adequacy in terms of partial structures fail. Indeed, the motivations for the generalizations in the partial structures approach are better met by the generalizations via approximation sets developed in "Generalizing Empirical Adequacy I" (Lutz in Synthese 191:3195-3225, 2014b. https://doi.org/10.1007/s11229-014-0440-3). Approximation sets also generalize partial structures.
\end{abstract}

Keywords Empirical adequacy · Constructive empiricism · Partial structures ·

Approximation sets · Approximation · Partial isomorphism · Partial homomorphism

\section{Introduction}

Van Fraassen's constructive empiricism, with its central notion of empirical adequacy, and the partial structures approach, with its central notions of partial truth and partial isomorphism, are two major lines of research in the semantic view, according to which scientific theories are best represented by model- or set-theoretic structures. Proponents of the partial structures approach have argued that these two approaches are a perfect fit for each other: Da Costa and French (1990) have discussed a means for describing empirical adequacy as partial truth, and Bueno (1997) has used the notions of partial structure, partial isomorphism, and partial truth for generalizing empirical adequacy.

I will argue that da Costa and French's attempt at describing empirical adequacy fails (Sect. 2), as does Bueno's attempt at generalizing empirical adequacy (Sect. 4). As shown in the first part of this discussion (Lutz 2014b), empirical adequacy is under certain conditions equivalent to the technical notion of generalized approximate truth, and it can be generalized with the help of approximation sets so that it can deal with situations involving lack of knowledge and approximations. I argue in this part of the

Sebastian Lutz

sebastian.lutz@gmx.net

1 Department of Philosophy, Uppsala University, Box 627, 75126 Uppsala, Sweden 
discussion that this generalization of empirical adequacy fits Bueno's motivations better than his own generalizations (Sect. 4). I will further show that the central concepts of the partial structures approach, partial truth (Sect. 2), partial isomorphism, and partial homomorphism ("Appendix A") can be captured in terms of approximation sets. Therefore everything that can be expressed with partial structures can be expressed with approximation sets. Indeed, approximation sets are more expressive than partial structures, which cannot express approximate functions nor approximate constants (Sect. 3).

The initial motivation for Bueno's generalizations was Suárez's criticism of constructive empiricism. I briefly show that both the criticism ("Appendix B") and the first of Bueno's replies (“Appendix C”) fail, while Bueno's second reply succeeds even against Suárez's unjustified criticism. This success, however, is the result of an almost trivially weak account of approximate empirical adequacy.

\section{Empirical adequacy as partial truth}

I will rely on the standard notations used in model theory (cf. Chang and Keisler 1990, $\S 1.3) .{ }^{1}$ A structure $\mathfrak{B}$ is a pair $\langle B, \mathscr{I}\rangle$ consisting of a domain $B$ and an interpretation function $\mathscr{I}$ from a set of $\lambda_{i}$-place relation symbols $R_{i}, \rho_{j}$-place function symbols $F_{j}$, and constant symbols $c_{k}$ to, respectively, $\lambda_{i}$-ary relations, $\rho_{j}$-ary functions, and constants on $B$. Unless stated otherwise, I will in the following always assume this set of symbols with the same arities. I will sometimes refer to symbols as 'terms' when this does not lead to ambiguity. $B$ will always be the domain $\operatorname{dom}(\mathfrak{B})$ of $\mathfrak{B}$, $C=\operatorname{dom}(\mathfrak{C})$ etc. If $\mathfrak{B}=\langle B, \mathscr{I}\rangle$, I write $R_{i}^{\mathfrak{B}}$ instead of $\mathscr{I}\left(R_{i}\right)$, and analogously for functions and constants. $R_{i}^{\mathfrak{C}}$ is the relation in $\mathfrak{C}$ that corresponds to relation $R_{i}^{\mathfrak{B}}$ in $\mathfrak{B}$, and analogously for functions and constants. In displayed form, I write a structure $\mathfrak{B}$ as $\left\langle B, R_{1}^{\mathfrak{B}}, \ldots, R_{s}^{\mathfrak{B}}, F_{1}^{\mathfrak{B}}, \ldots, F_{t}^{\mathfrak{B}}, c_{1}^{\mathfrak{B}}, \ldots, c_{u}^{\mathfrak{B}}\right\rangle$ or, for possibly infinite index sets, $\left\langle B, R_{i}^{\mathfrak{B}}, F_{j}^{\mathfrak{B}}, c_{k}^{\mathfrak{B}}\right\rangle_{i \in I, j \in J, k \in K}$. Substructures are defined as usual (see, e.g., Chang and Keisler 1990, pp. 21-22): A structure $\mathfrak{B}$ is a substructure of structure $\mathfrak{C}(\mathfrak{B} \subseteq \mathfrak{C}$ ) if and only if $B \subseteq C$, and each relation $R_{i}^{\mathfrak{B}}$ of $\mathfrak{B}$ is the restriction to $B$ of the corresponding relation $R_{i}^{\mathfrak{C}}$ of $\mathfrak{C}$ (i.e., $R_{i}^{\mathfrak{B}}=R_{i}^{\mathfrak{C}} \cap B^{\lambda_{i}}$ ), each function $F_{j}^{\mathfrak{B}}$ of $\mathfrak{B}$ is the restriction to $B$ of the corresponding function $F_{j}^{\mathfrak{C}}$ of $\mathfrak{C}$ (i.e., $F_{j}^{\mathfrak{B}}=F_{j}^{\mathfrak{C}} \mid B^{\rho_{j}}$ ), and each constant of $\mathfrak{B}$ is the corresponding constant of $\mathfrak{C}$ (cf. Hodges 1993, pp. 5-6).

Within this formalism, van Fraassen's definition of 'theory' (van Fraassen 1980, p. 64; cf. van Fraassen 2008, p. 238) can be expressed as follows:

Definition 1 A theory $\left\langle\left\{\mathfrak{T}_{n}\right\}_{n \in N},\left\{\mathbf{E}_{n}\right\}_{n \in N}\right\rangle$ contains a family, i.e. an indexed set, of structures (the models of the theory) and for each structure $\mathfrak{T}_{n}=\left\langle T_{n}, R_{i}^{\mathfrak{T}_{n}}\right.$, $\left.F_{j}^{\mathfrak{T}_{n}}, c_{k}^{\mathfrak{T}_{n}}\right\rangle_{i \in I_{n}, j \in J_{n}, k \in K_{n}}$ a set $\mathbf{E}_{n}$ of empirical substructures, such that for each $\mathfrak{E} \in \mathbf{E}_{n}$,

\footnotetext{
1 This choice of notation and the definitions are defended in the first part of this discussion (Lutz 2014b, $\S 2.1)$.
} 
$\mathfrak{E} \subseteq \mathfrak{T}_{n}$. With each model, a theory also contains every isomorphic structure and its corresponding ${ }^{2}$ empirical substructures.

The argument for this formalization is given in the first part of this discussion (Lutz $2014 b, \S \S 3.1-3.2)$, where it is shown in detail that a model of a theory can have multiple empirical substructures. ${ }^{3}$ As a shorthand, define $\left\langle\mathfrak{T}_{n}, \mathbf{E}_{n}\right\rangle:=\left\langle\left\{\mathfrak{T}_{n}\right\}_{n \in N},\left\{\mathbf{E}_{n}\right\}_{n \in N}\right\rangle$.

Van Fraassen's definition of 'appearances' (van Fraassen 1980, p. 64) can be expressed as follows:

Definition 2 Appearances are given by a set $\mathbf{A}$ of structures. A structure $\mathfrak{A} \in \mathbf{A}$ is an appearance.

Again, the first part of this discussion contains an argument for there being multiple appearances.

Definitions 1 and 2 then allow for

Definition 3 A theory $\left\langle\mathfrak{T}_{n}, \mathbf{E}_{n}\right\rangle$ is empirically adequate for appearances $\mathbf{A}$ if and only if there is some $n \in N$ such that for every $\mathfrak{A} \in \mathbf{A}$, there is an $\mathfrak{E} \in \mathbf{E}_{n}$ with $\mathfrak{E} \cong \mathfrak{A}$.

This is the precise formulation of the informal statement that "a theory is empirically adequate exactly if what it says about the observable things and events in this world, is true" (van Fraassen 1980, p. 12). And it is essential to go beyond this informal statement, since the notion of empirical adequacy "will have to be spelt out very carefully if it is not to bite the dust among hackneyed objections" (van Fraassen 1980, p. 19). It is with Definition 3, then, that van Fraassen (1980, p. 12, emphasis removed) defines constructive empiricism as the claim that science "aims to give us theories which are empirically adequate; and acceptance of a theory involves as belief only that it is empirically adequate".

Da Costa and French (1990, p. 257) now claim that van Fraassen's notion of empirical adequacy can be expressed in terms of partial truth as it is defined in the partial structures approach. The partial structures approach is motivated by a simple epistemological point: Most of the time, scientists do not have enough information about a domain to determine its structure with arbitrary precision. For most relations, it is at best known of some tuples of objects that they fall under the relation and known of some objects that they do not fall under it. For many if not most tuples this is unknown. Similarly, the value of a function is not known for all of its possible arguments. Partial structures are defined to take this lack of knowledge into account.

While most works on partial structures in the philosophy of science (e.g., Bueno 1997; da Costa and French 1990, 2000) do not consider functions, and the foundational paper by Mikenberg et al. (1986) does not consider constants, the respective definitions can be easily combined to give

\footnotetext{
${ }^{2}$ To be precise: If $f: T_{m} \longrightarrow T_{n}$ is an isomorphism between $\mathfrak{T}_{m}$ and $\mathfrak{T}_{n}$, then the set $\mathbf{E}_{n}$ of empirical substructures that corresponds to $\mathbf{E}_{m}$ contains all and only those structures $\mathfrak{E}$ for which there is an $\mathfrak{E}^{\prime} \in \mathbf{E}_{m}$ such that $f$ is an isomorphism between $\mathfrak{E}^{\prime}$ and $\mathfrak{E}$.

3 This was also confirmed by van Fraassen in private communication (Lutz 2014b, p. 3209, n. 9).
} 
Definition $4 \tilde{\mathfrak{B}}$ is a partial structure for the symbols $\left\{R_{i}, F_{j}, c_{k}\right\}_{i \in I, j \in J, k \in K}$ if and only if

$$
\tilde{\mathfrak{B}}=\left\langle B, R_{i}^{\tilde{\mathfrak{B}}}, F_{j}^{\tilde{\mathfrak{B}}}, c_{k}^{\tilde{\mathfrak{B}}}\right\rangle_{i \in I, j \in J, k \in K},
$$

where $B \neq \varnothing, R_{i}^{\tilde{\mathfrak{B}}}=\left\langle R_{i}^{\tilde{\mathfrak{B}},+}, R_{i}^{\tilde{\mathfrak{B}},-}, R_{i}^{\tilde{\mathfrak{B}}, \circ}\right\rangle$ is a tripartition of $B^{\lambda_{i}}$ for each $i \in I$, $F_{j}^{\tilde{\mathfrak{B}}}: W_{\tilde{\mathfrak{B}}, j} \longrightarrow B$ is a function with domain $W_{\tilde{\mathfrak{B}}, j} \subseteq B^{\rho_{j}}$ for each $j \in J$, and $c_{k}^{\tilde{\mathfrak{B}}} \in B$ for each $k \in K$.

The definition of partial structures by Mikenberg et al. (1986, def. 1) is recovered for $K=\varnothing$, the definition by da Costa and French (1990, pp. 255-256) and da Costa, Bueno, and French (1998, p. 605) for $J=\varnothing .{ }^{4}$ Lack of knowledge is represented by non-empty sets $R_{i}^{\tilde{\mathfrak{B}}, \circ}$ and sets $W_{\tilde{\mathfrak{B}}, j} \subset B^{\rho_{j}}$.

Taking into account background knowledge, expressed by a set $\tilde{\Pi}$ of sentences, the primary statements, Mikenberg et al. (1986, def. 2.ii) and da Costa and French (1990, p. 256) give

Definition 5 Structure $\mathfrak{C}$ is $\tilde{\mathfrak{B}}$-normal for primary statements $\tilde{\Pi}$ if and only if $C=B$, $R_{i}^{\tilde{\mathfrak{B}},+} \subseteq R_{i}^{\mathfrak{C}} \subseteq B^{\lambda_{i}}-R_{i}^{\tilde{\mathfrak{B}},-}$ for each $i \in I, F_{j}^{\mathfrak{C}} \mid W_{\tilde{\mathfrak{B}}, j}=F_{j}^{\tilde{\mathfrak{B}}}$ for each $j \in J$, $c_{k}^{\mathfrak{C}}=c_{k}^{\tilde{\mathfrak{B}}}$ for each $k \in K$, and $\mathfrak{C} \vDash \tilde{\Pi}$.

This allows for the definition of partial truth, also called 'pragmatic truth' or 'quasitruth':

Definition 6 Sentence $\varphi$ is partially true in partial structure $\tilde{\mathfrak{B}}$ relative to primary statements $\tilde{\Pi}$ if and only if there is a structure $\mathfrak{C}$ that is $\tilde{\mathfrak{B}}$-normal for $\tilde{\Pi}$ and $\mathfrak{C} \vDash \varphi$.

Since a theory may be given by a family $\left\{\mathfrak{T}_{n}\right\}_{n \in N}$ rather than a set of sentences, da Costa and French (1990, pp. 256-257) give ${ }^{5}$

Definition $7\left\{\mathfrak{T}_{n}\right\}_{n \in N}$ is partially true in partial structure $\tilde{\mathfrak{B}}$ relative to $\tilde{\Pi}$ if and only if for some $n \in N, \mathfrak{T}_{n}$ is $\tilde{\mathfrak{B}}$-normal for $\tilde{\Pi}$.

If thus $\left\{\mathfrak{T}_{n}: n \in N\right\}$ is the class of models of some finite set $\Sigma$ of sentences, $\left\{\mathfrak{T}_{n}\right\}_{n \in N}$ is partially true in $\tilde{\mathfrak{B}}$ relative to $\tilde{\Pi}$ if and only if the conjunction of the elements of $\Sigma$ is partially true in $\tilde{\mathfrak{B}}$ relative to $\tilde{\Pi}$.

Restricting their discussion to relational structures (which contain only relation symbols), da Costa and French (1990, p. 255) suggest formalizing our knowledge of a domain by first giving a partial structure $\tilde{\mathfrak{O}}=\left\langle O, R_{i}^{\tilde{\mathfrak{Q}}}\right\rangle_{i \in I}$ for the set of relation symbols $\left\{R_{i}\right\}_{i \in I}$, where $O$ is the set of observable objects. Then they extend the domain $O$ by the domain $U, O \cap U=\varnothing$, of unobservable objects and introduce

\footnotetext{
4 While da Costa and French (1990, p. 255) and da Costa et al. (1998, p. 605) define partial structures only for relations, their further definition of $\tilde{\mathfrak{B}}$-normal structures presumes that partial structures can contain constants as well.

5 Da Costa and French (1990) actually assume that a theory is given by a set T, rather than a family, of structures. Definition 7 is the result of the natural conversion of family $\left\{\mathfrak{T}_{n}\right\}_{n \in N}$ into the set $\left\{\mathfrak{T}_{n}: n \in N\right\}$. The inverse conversion goes from the set $\mathbf{T}$ to the family $\left\{\mathfrak{T}_{\mathfrak{T}}\right\}_{\mathfrak{T} \in \mathbf{T}}$. Note that these conversions do not provide a bijection between families and sets, since different families are converted into the same set.
} 
further relations $\left\{R_{i}\right\}_{i \in I^{\prime}}, I^{\prime} \cap I=\varnothing$ over $O \cup U$ to arrive at a partial structure $\tilde{\mathfrak{B}}=\left\langle O \cup U, R_{i}^{\tilde{\mathfrak{B}}}\right\rangle_{i \in I \cup I^{\prime}}\left(\right.$ da Costa and French 1990, p. 256). ${ }^{6}$ While they do not discuss explicitly what happens to the partial relations $\left\{R_{i}^{\tilde{\mathcal{O}}}\right\}_{i \in I}$ when the domain is extended, I take it that $R_{i}^{\tilde{\mathfrak{B}},+}=R_{i}^{\tilde{\mathcal{O}},+}$ and $R_{i}^{\tilde{\mathfrak{B}},-}=R_{i}^{\tilde{\mathfrak{O}},-}$ for each $i \in I$. In other words, the relation symbols $\left\{R_{i}\right\}_{i \in I}$ do not serve to describe the unobservable objects.

Da Costa and French (1990, p. 257) then suggest that $\tilde{\mathfrak{B}}$ and the primary statements "can be taken to represent [a theory's] 'empirical substructures', if [the primary statements are] restricted to observation statements only." And this then, they claim, "leads us to understand the "empirical adequacy' of a theory as its pragmatic truth" so that the formalism of partial structures "may provide a suitable theory of truth for van Fraassen's 'constructive empiricism"'. But it is at least not obvious how it could do this: For one, it is not clear in what sense $\tilde{\mathfrak{B}}$ "represents" the empirical substructures. As Bueno (1997, p. 595) furthermore points out, if it were clear, and empirical adequacy were then taken to be partial truth, it would follow that a theory is empirically adequate if and only if it is partially true according to its own empirical substructure. Thus the appearances would play no role whatsoever in the empirical adequacy of a theory.

It may be that da Costa and French (1990) had the following in mind: If the appearances $\mathbf{A}$ are but a singleton set $\{\mathfrak{A}\}$ with $\mathfrak{A}=\left\langle O, R_{i}^{\mathfrak{A}}\right\rangle_{i \in I}$ and $\left\langle\mathfrak{T}_{n}, \mathbf{E}_{n}\right\rangle$ is empirically adequate for $\{\mathfrak{A}\}$ (and hence contains the same predicates as $\mathfrak{A}$ ), then $\mathfrak{A}$ is isomorphic to an empirical substructure $\mathfrak{E} \in \mathbf{E}_{n}$ of $\mathfrak{T}_{n}$ for some $n \in N$. Since theories are closed under isomorphism, this is equivalent to $\mathfrak{A}$ being an empirical substructure $\mathfrak{E} \in \mathbf{E}_{m}$ of $\mathfrak{T}_{m}$ for some $m \in N$ (Hodges 1993, ex. 1.2.4b). Now $\tilde{\mathfrak{B}}:=\left\langle\operatorname{dom}\left(\mathfrak{T}_{m}\right),\left\langle R_{i}^{\mathfrak{A}}, O^{\lambda_{i}}-R_{i}^{\mathfrak{A}}, \operatorname{dom}\left(\mathfrak{T}_{m}\right)^{\lambda_{i}}-O^{\lambda_{i}}\right\rangle\right\rangle_{i \in I}$ is a partial structure, and $\mathfrak{T}_{m}$ is $\tilde{\mathfrak{B}}$-normal for any set $\tilde{\Pi}$ with $\mathfrak{T}_{m} \vDash \tilde{\Pi}$. Thus $\left\langle\mathfrak{T}_{n}, \mathbf{E}_{n}\right\rangle$ is partially true in partial structure $\tilde{\mathfrak{B}}$ relative to $\tilde{\Pi}$ and hence the empirical adequacy of a theory leads to its partial truth in a structure that corresponds to the appearance. Bueno's objection that the appearance plays no role in da Costa and French's attempt is thereby avoided, and it is clear in which sense the partial structure "represents" the appearance.

This result, however, does not show that van Fraassen's notion of empirical adequacy can be expressed as partial truth: Most obviously, the result relies on the assumption that the appearances have to be given by a single structure $\mathfrak{A}$, an assumption that van Fraassen decidedly does not share, as noted in connection with Definitions 1, 2 and 3 . Furthermore, the partial structure $\tilde{\mathfrak{B}}$ that corresponds to the appearance $\mathfrak{A}$ can only be constructed with the help of the model of the theory of which the appearance is a substructure. Thus $\tilde{\mathfrak{B}}$ is in part determined by the content of the theory (namely its claim about the cardinality of the domain). For van Fraassen (2008, p. 284), an

appearance is determined jointly by the measurement set-up (involving both apparatus and the system to which it is applied), the experimental practice, and the theoretical conceptual framework in which the target and measurement procedure are classified, characterized, and understood[,]

\footnotetext{
${ }^{6}$ Incidentally, the introduction of the nonobservational terms $\left\{R_{i}\right\}_{i \in I^{\prime}}$ amounts to a bipartition of the set of symbols akin to that in the Received View on scientific theories as developed by Carnap and Hempel.
} 
but not, it seems, additionally by the content of any particular theory. The difference between the theoretical conceptual framework and the content of a theory is crucial: The theoretical conceptual framework of a theory does not restrict the possible states of the world in any way and is neither true nor false. The content of a theory, on the other hand, does restrict the possible states of the world, and can therefore be false. ${ }^{7}$ What is worse, it is theoretical content that is needed for the construction of $\tilde{\mathfrak{B}}: \mathfrak{A}$ contains the empirical content of the theory; everything else, and thus specifically the cardinality of the theory's domain, is about unobservables. In his criticism of syntactic approaches, van Fraassen (1980, p. 54) states that the "empirical import of a theory cannot be isolated in this syntactical fashion, by drawing a distinction among theorems in terms of vocabulary" because "we shall be able to state in the observational vocabulary (however conceived) that there are unobservable entities, and, to some extent, what they are like." When expressing the appearance $\mathfrak{A}$ in its corresponding partial structure $\tilde{\mathfrak{B}}$, we shall be able to state in the partial structure that there are unobservable entities, and how many there are. For van Fraassen, this should be a big step in the wrong direction.

Finally, empirical adequacy has not been defined in terms of partial truth; rather, it has been shown that the empirical adequacy of a theory leads to its partial truth in a specifically constructed partial structure. But this partial structure can differ from theory to theory (since different theories can have domains of different cardinalities). Furthermore, the partial truth of the theory does not entail its empirical adequacy, that is, partial truth is only a necessary, not a sufficient condition for empirical adequacy. This is because the partial truth of $\left\langle\mathfrak{T}_{n}, \mathbf{E}_{n}\right\rangle$ in $\tilde{\mathfrak{B}}$ only entails that $\mathfrak{A}$ is a substructure of some $\mathfrak{T}_{m}, m \in N$. It does not entail that $\mathfrak{A}$ is an empirical substructure of $\mathfrak{T}_{m}$.

Thus even in this charitable reconstruction, da Costa and French's attempt to capture empirical adequacy in terms of partial truth is not successful.

\section{Generalizing partial structures}

While partial structures are not as hospitable to empirical adequacy as claimed by its proponents, the bigger problem is that they are also not particularly well-suited for their initially intended applications. It is probably because the discussions of partial structures and partial truth in the philosophy of science have typically ignored functions and, to some extent, constants, that this lack of applicability has so far been overlooked: Partial structures cannot capture the lack of knowledge about functions that typically occurs in the sciences, and cannot capture lack of knowledge about constants at all.

The interpretation of a relation symbol $R_{i}$ in a partial structure $\tilde{\mathfrak{B}}$ can capture fairly general cases of lack of knowledge by determining a set $R_{i}^{\tilde{\mathfrak{B}}, \circ}$ of tuples that may or may not be in the extension of $R_{i}$; however, in a partial structure each constant $c_{k}$ is interpreted by one unique element $c_{k}^{\tilde{\mathfrak{B}}} \in B$, so that the value of every constant is known precisely. Thus partial structures can be applied only in situations in which all constants are known with absolute precision, and thus almost only in situations

\footnotetext{
7 One could think of the difference as that between expressing the same proposition in different ways and expressing different propositions (cf. Eklund 2009, §2).
} 
in which no empirically determined constants like the fine structure constant, the gravitational constant, or any material constants occur at all. For instance, French and Ladyman (1997, p. 379) apply partial structures to the historical development of the Londons' equations for superconductivity, one of them relating the current density $\mathbf{j}$ and the magnetic field $\mathbf{H}$ via the speed of light $c$ and the constant $\Lambda=$ $\frac{m}{n_{\mathrm{s}} e^{2}}$, where $m$ is the mass and $e$ the charge of an electron, and $n_{\mathrm{S}}$ is the density of electrons participating in superconduction (French and Ladyman 1997, p. 373). All the constants occurring in this equation $\left(c, m, e\right.$, and $\left.n_{\mathrm{s}}\right)$ have to be assumed as known with absolute precision. This is especially problematic for the phenomenological constant $n_{\mathrm{s}}$, which is experimentally determined by, for instance, measuring the conductivity of superconductors for alternating currents (Annett 2004, p. 60).

Such a measurement will probably never be perfectly precise, and even if, in the far future, the electron density could be determined with perfect precision, this would not be relevant for the analysis of the historical developoment of the Londons' equation. Regarding fundamental constants like the fine structure constant, one may take the view that the presence of such a constant is an indicator that the theory in which they occur is not the final theory, which should be free of any constants. This view is at least controversial, since it is not obvious that there is such a theory; but the main problem is again that even if there is such a theory, its existence will make no difference to the applicability of partial structures before it has been found. In short, the preceding two replies to the partial structures' problem with constants assume that partial structures are only applied in a state of complete knowledge (about constant values or the final theory); but partial structures were intended to be applied (and have been applied by its main proponents) to situations with incomplete knowledge. Finally, one may take the view that all alleged constants in theories are actually functions, because their values change with time, energy scale, or in some other way. This view is also controversial, and also does not solve the problem for the application of partial structures to those theories that $d o$ involve constants (like the Londons' equation); furthermore, the treatment of functions in partial structures is barely less problematic than the treatment of constants. ${ }^{8}$

At first sight, partial structures seem to fare better with respect to function symbols, since they assign values to functions only over a restricted domain. But such a treatment of functions can capture only a lack of knowledge that is encountered very seldomly in the sciences. For in a partial structure $\tilde{\mathfrak{B}}$, the values of a function $F_{j}^{\tilde{\mathfrak{B}}}$ are known with arbitrary precision over $W_{\tilde{\mathfrak{B}}, j}$, but not at all over $B^{\rho_{j}}-W_{\tilde{\mathfrak{B}}, j}$. The result is that for each argument of the function, one either knows the value of the function with absolute precision, or has no information whatsoever about its possible values. In contrast, the measurement of, say, the time averaged intensity $\bar{\psi}$ of a light wave over some spatial interval $\left[x_{1}, x_{2}\right]$ will typically have a finite precision, giving a range of possible intensity values $\left[y_{1}, y_{2}\right] \subset \mathbb{R}^{\geq 0}$ for each point $x \in\left[x_{1}, x_{2}\right]$. A partial structure, however, can only capture measurements that, at any point $x$, give either a precise value $\bar{\psi}(x)=y_{3} \in \mathbb{R}^{\geq 0}$, or the trivial range $\mathbb{R}$. In the case of the Londons' equation discussed by French and Ladyman (1997, p. 379), j and H are vec-

\footnotetext{
8 I owe these objections to the relevance of partial structures' problem with constants to an anonymous reviewer.
} 
tor valued functions over space and time. In a partial structure, these function values have to be absolutely precise over one (possibly empty, possibly all-encompassing) space-time volume and absolutely imprecise over the remainder. Over no space-time value can they be determined only up to a non-zero, finite interval. Similarly, if the fine-structure constant, for instance, is actually a function over the energy scale, partial structures could only render it completely undetermined at some energies, and determined with absolute precision at the remaining energies. Our current knowledge of the fine-structure constant as being within in some non-zero, finite interval could not be expressed.

Thus partial structures are ill-equipped to capture lack of knowledge about functions and constants, and accordingly also ill-equipped to capture approximations of constants and functions. It is possible to reformulate a theory so that it contains relations instead of functions and constants. However, this increases the number of axioms of the theory, complicates the application of partial structures in a way analogous to the way that it complicates the application of standard structures (Hodges 1993, p. 2 ), and removes partial structures further from actual scientific practice. Besides, if theories need to be reformulated so that they can be analyzed with partial structures, the partial structures approach becomes in a sense superfluous: Every analysis of a theory in terms of partial structures is equivalent to an analysis of a reformulated theory in standard model theory (Lutz 2015). And if a theory has to be reformulated for an analysis in terms of partial structures, it might just as well be reformulated for an analysis in terms of standard model theory.

As argued in the first part of this discussion (Lutz 2014b, §§5.2-5.3), imprecise functions and constants can be handled fairly satisfyingly with the help of approximation sets, which are sets of structures that are generated from the denotations of approximate relation, function, and constant symbols. The denotation of a $\lambda_{i}$-place relation symbol $R_{i}$ that is approximate over some domain $A$ tripartitions the product domain $A^{\lambda_{i}}$ into a set $R_{i}^{+}$of definite instances (the positive extension of $R_{i}$ ), a set $R_{i}^{-}$of definite non-instances (the negative extension), and a set $R^{\circ}$ of borderline cases of $R_{i}$ (the neutral extension). The denotation of a function symbol $F_{j}$ that is approximate over $A$ does not assign a single element $b \in A$ to a $\rho_{j}$-tuple $\left\langle a_{1}, \ldots, a_{\rho_{j}}\right\rangle \in A^{\rho_{j}}$, but rather a set $F_{j}^{+\circ}\left(a_{1}, \ldots, a_{\rho_{j}}\right) \subseteq A$. I will refer to the set $\left\{\left\langle a_{1}, \ldots, a_{\rho_{j}}, b\right\rangle: a_{1}, \ldots, a_{\rho_{j}} \in A, b \in F_{j}^{+\circ}\left(a_{1}, \ldots, a_{\rho_{j}}\right)\right\}$ as the non-negative extension $F^{+\circ}$ of $F_{j}$. If $F_{j}^{+\circ}\left(a_{1}, \ldots, a_{\rho_{j}}\right)$ is a singleton set, I will say that $F_{j}$ has a positive extension for $\left\langle a_{1}, \ldots, a_{\rho_{j}}\right\rangle$. Considering constant symbols 0 -place function symbols, this means that the denotation of a constant symbol $c_{k}$ that is approximate over $A$ is a set $c_{k}^{+\circ} \subseteq A$.

The denotations of approximate terms over $A$ lead to a set of of structures (Lutz 2014b, def. 7), which can be restricted to a penumbral set $\mathbf{P}$ to express additional information not expressed by the denotations of the terms. Fine (1997, p. 123) gives the example of 'pink' and 'red' having disjoint extensions even if the positive extension of one is not a subset of the negative extension of the other. Przełęcki (1976, p. 376) gives the example of a function symbol's extensions, which may be restricted "by some additional conditions of a more theoretical nature", demanding, for instance, that any extension of the symbol be continuous. 
Definition 8 Let the terms $\left\{R_{i}, F_{j}, c_{k}\right\}_{i \in I, j \in J, k \in K}$ be approximate over domain $A$ with positive, negative, and non-negative extensions $\left\{R_{i}^{+}, R_{i}^{-}, F_{j}^{+\circ}, c_{k}^{+\circ}\right\}_{i \in I, j \in J, k \in K}$, and penumbral set $\mathbf{P}$. Then the terms' approximation set $\mathbf{M}$ for $A$ contains all and only structures $\mathfrak{M} \in \mathbf{P}$ for which

$$
\begin{gathered}
M=A, \\
R_{i}^{+} \subseteq R_{i}^{\mathfrak{M}} \subseteq A^{\lambda_{i}}-R_{i}^{-} \text {for all } i \in I, \\
F_{j}^{\mathfrak{M}} \subseteq F_{j}^{+\circ} \text { for all } j \in J, \text { and } \\
c_{k}^{\mathfrak{M}} \in c_{k}^{+\circ} \text { for all } k \in K
\end{gathered}
$$

The penumbral set is assumed to be such that $R_{i}^{+}=\bigcap\left\{R_{i}^{\mathfrak{M}}: \mathfrak{M} \in \mathbf{M}\right\}, R_{i}^{-}=$ $\bigcap\left\{M^{\lambda_{i}}-R_{i}^{\mathfrak{M}}: \mathfrak{M} \in \mathbf{M}\right\}, F_{j}^{+\circ}=\bigcup\left\{F_{j}^{\mathfrak{M}}: \mathfrak{M} \in \mathbf{M}\right\}$, and $c_{k}^{+\circ}=\bigcup\left\{c_{k}^{\mathfrak{M}}: \mathfrak{M} \in \mathbf{M}\right\}$. In other words, the penumbral set is assumed to exclude only structures from the approximation set that cannot be excluded with the help of positive, negative, and non-negative extensions. Note that condition (4) for functions is equivalent to the demand that for each $\rho_{j}$-tuple $\left\langle a_{1}, \ldots, a_{\rho_{j}}\right\rangle \in A^{\rho_{j}}$ of the domain, $F_{j}^{\mathfrak{M}}\left(a_{1}, \ldots, a_{\rho_{j}}\right) \in$ $F_{j}^{+\circ}\left(a_{1}, \ldots, a_{\rho_{j}}\right)$.

Using approximation sets instead of partial structures does not lead to any loss of expressive power, because every partial structure can be expressed with the help of a corresponding approximation set:

Definition 9 Let $\tilde{\mathfrak{B}}=\left\langle B, R_{i}^{\tilde{\mathfrak{B}}}, F_{j}^{\tilde{\mathfrak{B}}}, c_{k}^{\tilde{\mathfrak{B}}}\right\rangle_{i \in I, j \in J, k \in K}$ be a partial structure, $\tilde{\Pi}$ a set of primary statements, and let $\mathbf{P}$ be the class of models of $\tilde{\Pi}$. Then the approximation set for $\left\{R_{i}^{+}, R_{i}^{-}, F_{j}^{+\circ}, c_{k}^{+\circ}\right\}_{i \in I, j \in J, k \in K}$ over $B$ with penumbral set $\mathbf{P}$ and $R_{i}^{+}=R_{i}^{\tilde{\mathfrak{B}},+}$, $R_{i}^{-}=R_{i}^{\tilde{\mathfrak{B}},-}, F_{j}^{+\circ}=F_{j}^{\tilde{\mathfrak{B}}} \cup\left(B^{\rho_{j}}-W_{\tilde{\mathfrak{B}}, j}\right) \times B$, and $c_{k}^{+\circ}=\left\{c_{k}^{\tilde{\mathfrak{B}}}\right\}$ for $i \in I, j \in$ $J, k \in K$ corresponds to $\tilde{\mathfrak{B}}$ and $\tilde{\Pi}$.

Claim 1 For any partial structure $\tilde{\mathfrak{B}}$, the set of $\tilde{\mathfrak{B}}$-normal structures relative to $\tilde{\Pi}$ is the corresponding approximation set.

Proof ' $\Rightarrow$ ': Let $\mathfrak{C}$ be $\tilde{\mathfrak{B}}$-normal for $\tilde{\Pi}$. Then $C=B$ and $\mathfrak{C} \in \mathbf{P}$. Furthermore, $R_{i}^{+}=$ $R_{i}^{\tilde{\mathfrak{B}},+} \subseteq R_{i}^{\mathfrak{C}} \subseteq B^{\lambda_{i}}-R_{i}^{\mathfrak{B},-}=B^{\lambda_{i}}-R_{i}^{-}$for each $i \in I, F_{j}{ }^{\mathfrak{C}} \mid W_{\tilde{\mathfrak{B}}, j}=F_{j}^{\tilde{\mathfrak{B}}}$ so that $F_{j}^{\mathfrak{C}} \subseteq F_{j}^{\tilde{\mathfrak{B}}} \cup\left(B^{\rho_{j}}-W_{\tilde{\mathfrak{B}}, j}\right) \times B=F_{j}^{+\circ}$ for each $j \in J$, and $c_{k}^{\mathfrak{C}}=c_{k}^{\tilde{\mathfrak{B}}} \in\left\{c_{k}^{\tilde{\mathfrak{B}}}\right\}=c_{k}^{+\circ}$ for each $k \in K$. Thus $\mathfrak{C}$ is in the approximation set corresponding to $\tilde{\mathfrak{B}}$ and $\tilde{\Pi}$.

' $\Leftarrow$ ': Let $\mathfrak{C}$ be in the approximation set for $\left\{R_{i}^{+}, R_{i}^{-}, F_{j}^{+\circ}, c_{k}^{+\circ}\right\}_{i \in I, j \in J, k \in K}$ over $B$ with penumbral set $\mathbf{P}$. Then $C=B$ and $\mathfrak{C} \vDash \tilde{\Pi}$. Furthermore, $R_{i}^{\tilde{\mathfrak{B}},+}=R_{i}^{+} \subseteq R_{i}^{\mathfrak{C}} \subseteq$ $B^{\lambda_{i}}-R_{i}^{+}=B^{\lambda_{i}}-R_{i}^{\mathfrak{B},-}$ for each $i \in I, F_{j}^{\mathfrak{B}} \subseteq F_{j}^{+\circ}=F_{j}^{\tilde{\mathfrak{B}}} \cup\left(B^{\rho_{j}}-W_{\tilde{\mathfrak{B}}, j}\right) \times B$ so that $F_{j}{ }^{\mathfrak{C}} \mid W_{\tilde{\mathfrak{B}}, j}=F_{j}^{\tilde{\mathfrak{B}}}$, and $c_{k}^{\mathfrak{C}} \in c_{k}^{+\circ}=\left\{c_{k}^{\tilde{\mathfrak{B}}}\right\}$ so that $c_{k}^{\mathfrak{C}}=c_{k}^{\tilde{\mathfrak{B}}}$. Therefore $\mathfrak{C}$ is $\tilde{\mathfrak{B}}$-normal for $\tilde{\Pi}$.

If the approximation set corresponding to $\tilde{\mathfrak{B}}$ and $\tilde{\Pi}$ is a singleton set $\{\mathfrak{C}\}$, then I will say that $\mathfrak{C}$ corresponds to $\tilde{\mathfrak{B}}$ and $\tilde{\mathfrak{B}}$ corresponds to $\mathfrak{C}$. 
Since not every approximation set corresponds to a partial structure, the notion of an approximation set is a proper generalization of the notion of a partial structure. In fact, the notion of an approximation set generalizes the notion of a partial structure in a way that solves the latter's problems with constants and functions: In a partial structure's corresponding approximation set the non-negative extension of a constant $c_{k}$ is $c_{k}^{+\circ}=\left\{c_{k}^{\tilde{\mathfrak{B}}}\right\}$, that is, $c_{k}$ has a (unique) positive extension, and every structure in the approximation set assigns the same object to $c_{k}$. By contrast, an approximation set in general allows any kind of non-negative extension, and thus can contain structures that assign different objects to $c_{k}$. The situation is analogous for function symbols $F_{j}$ : In a partial structure's corresponding approximation set, the non-negative extension $F_{j}^{+\circ}=F_{j}^{\tilde{\mathfrak{B}}} \cup\left(B^{\rho_{j}}-W_{\tilde{\mathfrak{B}}, j}\right) \times B$ takes a unique value for any argument from $W_{\tilde{\mathfrak{B}}, j}$ and takes the whole domain $B$ as value for any argument from $B^{\rho_{j}}-W_{\tilde{\mathfrak{B}}, j}$. An approximation set, on the other hand, allows for a non-negative extension that is, for some or all of the arguments of $F_{j}$, neither unique nor completely unrestricted. In other words, for each argument of $F_{j}$, there is some set of values that $F_{j}$ is assigned by the structures in the approximation set, and this set of values may be any subset of the domain. Thus approximation sets can capture both imprecise values for constants and imprecise values for functions. ${ }^{9}$

Using approximation sets, one can define the approximate truth of a class $\mathbf{E}$ and a family $\left\{\mathfrak{T}_{n}\right\}_{n \in N}$ of structures:

Definition 10 Let $\mathbf{M}$ be an approximation set. A class of structures $\mathbf{E}$ is approximately true in $\mathbf{M}$ if and only if for some $\mathfrak{E} \in \mathbf{E}$ and some $\mathfrak{M} \in \mathbf{M}, \mathfrak{E}=\mathfrak{M} .{ }^{10} \mathrm{~A}$ family $\left\{\mathfrak{T}_{n}\right\}_{n \in N}$ is approximately true in $\mathbf{M}$ if and only if $\left\{\mathfrak{T}_{n}: n \in N\right\}$ is approximately true in $\mathbf{M}$.

The relation between partial truth and approximate truth is given by

Claim $2\left\{\mathfrak{T}_{n}\right\}_{n \in N}$ is partially true in partial structure $\tilde{\mathfrak{B}}$ for $\tilde{\Pi}$ if and only if $\left\{\mathfrak{T}_{n}\right\}_{n \in N}$ is approximately true in the corresponding approximation set.

Proof Immediately from Claim 1.

Therefore approximate truth is a generalization of partial truth. Note that if $\left\{\mathfrak{T}_{n}: n \in\right.$ $N\}$ is the class of models of a set of sentences $\Sigma$ and the partial structure $\tilde{\mathfrak{B}}$ corresponds to a structure $\mathfrak{B}$, the partial truth of $\left\{\mathfrak{T}_{n}\right\}_{n \in N}$ in $\tilde{\mathfrak{B}}$ is equivalent to the truth of $\Sigma$ in $\mathfrak{B}$.

Approximation sets generalize partial structures, approximate truth generalizes partial truth, and partial isomorphisms (Definition 12) and partial homomorphisms (Definition 20) can be expressed in terms of isomorphisms and homomorphisms between the elements of approximation sets ("Appendix A"). Hence the partial structures approach can be completely captured and generalized in terms of approximation sets, which has a convenient corollary: Every possible application of partial structures is also a possible application of approximation sets. Specifically, any application of

\footnotetext{
9 The first part of this discussion contains a fairly detailed analysis of approximation sets for the intensity of a light wave.

10 Given van Fraassen's formalism, one might have expected an isomorphism at this point, $\mathfrak{E} \cong \mathfrak{M}$. This option will be discussed below under the moniker 'approximate truth up to isomorphism' (Definition 15).
} 
partial structures can be translated into an application of their corresponding approximation sets. For the reasons given above, the converse is not true. ${ }^{11}$

\section{Generalizing empirical adequacy}

Bueno (1997, p. 595) suggests using the partial structures approach not for capturing, but rather for "extending van Fraassen's proposals within a partial structure conception". While his two generalizations are meant to allow for the formalization of common scientific practices within constructive empiricism and have been the basis of further development and applications of the partial structures approach, they have initially been suggested as a response to a criticism of empirical adequacy by Suárez (1995, 2005). I argue in "Appendix B" that this initial motivation is not relevant because Suárez's criticism fails.

Both of Bueno's generalizations rely on the idea that the appearances are seldom known well enough to be described in a set $\mathbf{A}$ of structures, and that usually a set of partial structures must suffice, leading to the partial appearances $\tilde{\mathbf{A}}$. This is more than just a formal modification of the notion of empirical adequacy: Van Fraassen is arguably justified in assuming that the appearances are represented by structures, because a theory has to be empirically adequate with respect to all the appearances, known in all precision (cf. Monton and Mohler 2008, §1.5). While van Fraassen's notion of empirical adequacy thus relies exclusively on what the appearances are like, Bueno's notion includes our lack of knowledge about them. Bueno thus gives a generalization of empirical adequacy that takes epistemic states into account, and should therefore be equivalent to van Fraassen's notion in the special case that there is no epistemic uncertainty about the phenomena. I will argue in the sequel that both of Bueno's generalizations of empirical adequacy are inadequate, and that his intent is better captured by the generalizations of empirical adequacy suggested in the first part of this discussion.

\subsection{Partial empirical adequacy}

Bueno's first suggested generalization can be motivated by the simple observation that if the appearances are given by partial structures, there can be no isomorphisms between the appearances and the empirical substructures of any theory, because substructures and isomorphisms are defined only for structures. Bueno (1997, p. 596) therefore defines the partial substructures of a relational partial structure, which can easily be generalized to partial structures as follows:

Definition $11 \tilde{\mathfrak{B}}$ is a partial substructure of partial structure $\tilde{\mathfrak{C}}, \tilde{\mathfrak{B}} \subseteq \tilde{\mathfrak{C}}$, if and only if $B \subseteq C, R_{i}^{\tilde{\mathfrak{B}},+}=R_{i}^{\tilde{\mathfrak{C}},+} \cap B^{\lambda_{i}}, R_{i}^{\tilde{\mathfrak{B}},-}=R_{i}^{\tilde{\mathfrak{C}},-} \cap B^{\lambda_{i}}, R_{i}^{\tilde{\mathfrak{B}}, \circ}=R_{i}^{\tilde{\mathfrak{C}}, \circ} \cap B^{\lambda_{i}}$ for all $i \in I, F_{j}^{\tilde{\mathfrak{B}}}=F_{j}^{\tilde{\mathfrak{C}}} \mid B^{\rho_{j}}$ for all $j \in J$, and $c_{k}^{\tilde{\mathfrak{B}}}=c_{k}^{\tilde{\mathcal{C}}}$ for all $k \in K$.

\footnotetext{
${ }^{11}$ For instance, the analysis of the intensity of a light wave contained in the first part of this discussion has no counterpart in the partial structures approach.
} 
It is easy to see that if $\mathfrak{B}$ corresponds to $\tilde{\mathfrak{B}}$ and $\mathfrak{C}$ corresponds to $\tilde{\mathfrak{C}}$, then $\tilde{\mathfrak{B}} \subseteq \tilde{\mathfrak{C}}$ if and only if $\mathfrak{B} \subseteq \mathfrak{C}$.

Further, Bueno defines a partial isomorphism between two relational partial structures, which can also easily be generalized to partial structures:

Definition $12 f: B \longrightarrow C$ is a partial isomorphism between partial structures $\mathfrak{B}$ and $\mathfrak{C}$ if and only if $f$ is a bijection and it holds that $f\left(R_{i}^{\tilde{\mathfrak{B}},+}\right)=R_{i}^{\tilde{\mathfrak{C}},+}$ and $f\left(R_{i}^{\tilde{\mathfrak{B}},-}\right)=$ $R_{i}^{\tilde{\mathfrak{C}},-}$ for each $i \in I, f\left(W_{\tilde{\mathfrak{B}}, j}\right)=W_{\tilde{\mathfrak{C}}, j}$ and $f\left(F_{j}^{\tilde{\mathfrak{B}}} x_{1} \ldots x_{\rho_{j}}\right)=F_{j}^{\tilde{\mathfrak{C}}} f\left(x_{1}\right) \ldots f\left(x_{\rho_{j}}\right)$ for each $x_{1}, \ldots, x_{\rho_{j}} \in W_{\tilde{\mathfrak{B}}, j}, j \in J$, and $f\left(c_{k}^{\tilde{\mathfrak{B}}}\right)=c_{k}^{\tilde{\mathfrak{C}}}$ for each $k \in K .^{12}$

$\tilde{\mathfrak{B}}$ and $\tilde{\mathfrak{C}}$ are called partially isomorphic $(\tilde{\mathfrak{B}} \cong \tilde{\mathfrak{C}})$ if and only if there is a partial isomorphism between $\tilde{\mathfrak{B}}$ and $\tilde{\mathfrak{C}}{ }^{13}$ It is easy to see that if $\mathfrak{B}$ corresponds to $\tilde{\mathfrak{B}}$ and $\mathfrak{C}$ corresponds to $\tilde{\mathfrak{C}}$, then $\tilde{\mathfrak{B}} \cong \tilde{\mathfrak{C}}$ if and only if $\mathfrak{B} \cong \mathfrak{C}$. Since the notion of partial isomorphism has been the basis of much further development in the partial structure approach (e.g., Bueno 1999, pp. 65-66; French 2000, p. 106; Bueno 2000, §3.2, §4.1; Bueno et al. 2002, p. 499; da Costa and French 2003, pp. 48-52; French 2003, p. 1480; Bueno and Colyvan 2011, p. 14) I show in "Appendix A" that the concept has an analogue for approximation sets.

Bueno (1997, p. 596) further assumes that theories are given as families $\left\{\tilde{\mathfrak{T}}_{n}\right\}_{n \in N}$ of partial structures, with a set $\tilde{\mathbf{E}}_{n}$ of partial empirical substructures for each $n \in N .{ }^{14}$ But this assumptions is problematic: In constructive empiricism, theories and their empirical substructures are assumed to be given by normal structures, not partial structures, and Tarski semantics only provides a way of translating the equations of a theory (like Maxwell's equations and the Londons' equations) into normal structures. ${ }^{15}$ And it is not obvious how a theory $\left\langle\mathfrak{T}_{n}, \mathbf{E}_{n}\right\rangle$ given by normal structures can be described as a set of partial structures. Of course, every normal structure corresponds to a partial structure, but in this identification partial truth, partial substructures, and partial isomorphisms amount to truth, substructures, and isomorphisms, respectively, so nothing is gained.

However, there may be a solution to this problem: Given $\left\langle\mathfrak{T}_{n}, \mathbf{E}_{n}\right\rangle$, one can try finding a set $M \subseteq \wp N$ of subsets of $N$ such that for each $m \in M,\left\{\mathfrak{T}_{n}: n \in m\right\}$ is an approximation set corresponding to a partial structure $\tilde{\mathfrak{T}}_{m}$ and $\bigcup_{n \in m} \mathbf{E}_{n}$ is a union of approximation sets that correspond to elements of a set $\tilde{\mathbf{E}}_{m}$ of partial structures. This provides a partial theory $\left\langle\left\{\tilde{\mathfrak{T}}_{m}\right\}_{m \in M},\left\{\tilde{\mathbf{E}}_{m}\right\}_{m \in M}\right\rangle$. Any theory with approximation claims about relations and functions (of the kind that can be captured by partial structures) leads in this way to non-trivial partial structures. As a toy theory, consider $\forall x(P x \rightarrow Q x) \wedge \forall x(Q x \rightarrow R x)$. For fixed $P^{\mathfrak{B}}$ and $R^{\mathfrak{B}}$ with

\footnotetext{
12 For a relation $R_{i}, \quad f\left(R_{i}\right)$ is the image of $R_{i}$ under $f$, that is, $f\left(R_{i}\right)=$ $\left\{\left\langle f\left(x_{1}\right), \ldots, f\left(x_{\lambda_{i}}\right)\right\rangle: R_{i} x_{1} \ldots x_{\lambda_{i}}\right\}$.

13 The concept given by Definition 12 must not be confused with the concept that French and Ladyman (1997, p. 371, n. 16) call 'partial isomorphism', the isomorphism between substructures (cf. Ebbinghaus et al. 1994, def 1.1).

14 Bueno $(2000, \S 3.2)$ relies on this assumption as well.

15 Suárez and Cartwright (2008, p. 74) encounter the same problem when discussing partial isomorphisms involving theories.
} 
$P^{\mathfrak{B}} \subset R^{\mathfrak{B}}$, it leads to the non-trivial partial structure $\tilde{\mathfrak{B}}=\left\langle B, P^{\tilde{\mathfrak{B}}}, Q^{\tilde{\mathfrak{B}}}, R^{\tilde{\mathfrak{B}}}\right\rangle$ with $Q^{\tilde{\mathfrak{B}}, \circ}=B-\left(P^{\mathfrak{B}} \cup Q^{\mathfrak{B}}\right) \neq \varnothing .{ }^{16}$

Using the notions of partial substructure and partial isomorphism and the assumption of partial theories, Bueno (1997, p. 596) introduces

Definition 13 A partial theory $\left\langle\left\{\tilde{\mathfrak{T}}_{m}\right\}_{m \in M},\left\{\tilde{\mathbf{E}}_{m}\right\}_{m \in M}\right\rangle$ is partially empirically adequate for the partial appearances $\tilde{\mathbf{A}}$ if and only if there is some $m \in M$ such that for every $\tilde{\mathfrak{A}} \in \tilde{\mathbf{A}}$, there is an $\tilde{\mathfrak{E}} \in \tilde{\mathbf{E}}_{m}$ with $\tilde{\mathfrak{E}} \cong \tilde{\mathfrak{A}}$.

As noted, capturing the appearances as a set of partial structures rather than a set of structures introduces an epistemic element into empirical adequacy. If there is no lack of knowledge about the appearances, then $R_{i}^{\tilde{\mathfrak{B}}, \circ}=\varnothing$ and $W_{\tilde{\mathfrak{B}}, j}=A^{\rho_{j}}$ for all $i \in I, j \in J$, and partial appearances $\tilde{\mathfrak{A}}$, so that there are structures corresponding to the partial structures. Since furthermore partial substructures and partial isomorphisms generalize substructures and isomorphisms, Definition 13 generalizes Definition 3 of empirical adequacy to partial theories and partial appearances. Therefore, for full information, partial empirical adequacy is equivalent to empirical adequacy, as required.

Since Suárez's criticism fails, it is not important that partial empirical adequacy cannot cope with Suárez's criticism ("Appendix C.1"). A real problem is that almost no theory is ever partially empirically adequate, and if so, only for a very short time (with some notable exceptions). This is because the partial appearances are meant to represent our lack of knowledge of the appearances. And to be partially empirically adequate, the partial theory must have a partial empirical substructure that is partially isomorphic to the partial appearances, and therefore describe our state of knowledge about the appearances. Formally, for each relation $R_{i}^{\tilde{\mathfrak{A}}}$ of a partial appearance, there has to be a relation $R_{i}^{\tilde{E}}$ of a partial empirical substructure of the partial theory such that a partial isomorphism maps all and only elements in $R_{i}^{\tilde{\mathcal{A}}, \circ}$ to elements in $R_{i}^{\tilde{\mathfrak{E}}, \circ}$. Therefore, if a theory asserts more about any appearance than what is currently known (so that $R_{i}^{\tilde{\mathfrak{A}}, \circ}$ is mapped to a proper subset of $R_{i}^{\tilde{\mathbb{E}}, \circ}$ ), it is not partially empirically adequate. If a theory asserts in some respects more about any appearance than what is currently known about any appearance and in some respects asserts less (so that $R_{i}^{\tilde{\mathfrak{A}} \text {, }}$ is mapped neither to a subset nor a superset of $R_{i}^{\tilde{\mathfrak{E}}, \circ}$ ), it is not partially empirically adequate either. This point becomes even clearer when considering Bueno's claim that partial isomorphisms to partial substructures amount to "approximate embeddings" (Bueno 1997, p. 597): If the partial appearances are meant to describe the approximation with which we know the appearances, then a partial theory is partially empirically adequate only if for some $n \in N$, the partial empirical substructures in $\tilde{\mathbf{E}}_{n}$ contain the exact approximations up to which we know the appearances. Therefore, if a partial

\footnotetext{
${ }^{16}$ Suárez and Cartwright (2008, p. 75) instead suggest introducing additional relations that are partial. But this does not solve the current problem of the original relations (e. g., $Q$ ) not being partial. Bueno (personal communication, 30 September 2011) has suggested that partial structures describe the scientists' uncertainty about specific aspects of a theory. For example, the accuracy of a theory may be known only up to a certain approximation. In this case, however, partial structures do not formalize theories, but scientists' beliefs, and a theory would have to be formalized differently depending on how much of it is accepted in the scientific community. This is not how, e. g., French and Ladyman (1997) apply partial structures to theories.
} 
theory describes the appearances up to some approximation, the theory is not partially empirically adequate until we have determined the appearances with exactly that approximation. Of course, if later the we know a little bit more, the theory ceases to be partially empirically adequate. ${ }^{17}$

The concept of approximate empirical adequacy defined in the first part of this discussion (Lutz 2014b, def. 10, 13) avoids these problems by using, instead of a set $\tilde{\mathbf{A}}$ of partial structures, a set $\mathbf{A}$ of approximation sets for the appearances (called 'approximate appearances'). As argued in Sect. 3, this also generalizes the kind of approximations for functions and constants that can be captured in partial appearances.

Definition 14 Given the approximate appearances $\mathbf{A}$, a theory $\left\langle\mathfrak{T}_{n}, \mathbf{E}_{n}\right\rangle$ is approximately empirically adequate for $\mathbf{A}$ if and only if there is some $n \in N$ such that for every $\mathbf{Q} \in \mathbf{A}$, there are an $\mathfrak{A} \in \mathbf{Q}$ and an $\mathfrak{E} \in \mathbf{E}_{n}$ with $\mathfrak{E} \cong \mathfrak{A}$.

This expresses van Fraassen's assumption that a theory is empirically adequate if and only if "what the theory says about what is observable (by us) is true" (van Fraassen 1980 , p. 18, emphasis removed) if truth is taken to be truth up to isomorphism:

Definition 15 Let $\mathbf{M}$ be an approximation set. A class of structures $\mathbf{E}$ is approximately true up to isomorphism in $\mathbf{M}$ if and only if for some $\mathfrak{E} \in \mathbf{E}$ and some $\mathfrak{M} \in \mathbf{M}, \mathfrak{E} \cong \mathfrak{M}$.

The difference to Definition 10 stems from the difference between the notion of partial truth (Definition 7), which relies on the identity of structures, and van Fraassen's notion of empirical adequacy (that is, the truth of the empirical part of a theory), which relies on isomorphism. ${ }^{18}$ This difference turns out to be crucial considering Bueno's dismissal of the following suggestion for generalizing the empirical adequacy of a theory $\left\langle\mathfrak{T}_{n}, \mathbf{E}_{n}\right\rangle$ : One could demand that there be some $n \in N$ such that for every $\tilde{\mathfrak{A}} \in$ $\tilde{\mathbf{A}}$, there is an $\tilde{\mathfrak{A}}$-normal structure $\mathfrak{A}$ with $\mathfrak{A} \in \mathbf{E}_{n}$. However, because of the definition of ' $\tilde{\mathfrak{A}}$-normal structure', this would require that the empirical substructures in $\mathbf{E}_{n}$ have the same domains as the partial appearances. The problem with this requirement, so Bueno (1997, p. 594), is that in practice "we would hardly, if ever, see such a constraint satisfied". Since the identity of empirical substructures and appearances is Bueno's sole stated reason for rejecting this generalization, Definition 14 should be acceptable, because it avoids exactly this identity:

Claim 3 If each approximate appearance in the approximate appearances $\mathbf{A}$ correspond to a partial appearance in the partial appearances $\tilde{\mathbf{A}}$ and vice versa, a theory $\left\langle\mathfrak{T}_{n}, \mathbf{E}_{n}\right\rangle$ is approximately empirically adequate for $\mathbf{A}$ if and only if there is some

\footnotetext{
17 Similar criticisms apply to the applications of the notion of partial isomorphism by Bueno (1999, p. 67), French (2000, pp. 110-111), Bueno (2000, §3.2), da Costa and French (2003, pp. 73, 102, 150, 193), French (2003, §4-5), and Bueno and Colyvan (2011, §5.3). If partial structures are taken to formalize scientists' beliefs (see n. 16), a theory that is not partially empirically adequate in light of new appearances may subsequently become partially empirically adequate simply because scientists change their beliefs according to the new appearances. In this case, partial empirical adequacy could be seen as a criterion for the adequacy of scientists beliefs. Specifically, a completely false theory may be partially empirically adequate if everyone is uncertain about its truth.

18 In the first part of this discussion (Lutz 2014b, def. 7), 'truth' is defined in the way that 'truth up to isomorphism' is defined here.
} 
$n \in N$ such that for every $\tilde{\mathfrak{A}} \in \tilde{\mathbf{A}}$, there are an $\tilde{\mathfrak{A}}$-normal structure $\mathfrak{A}$ and an $\mathfrak{E} \in \mathbf{E}_{n}$ with $\mathfrak{E} \cong \mathfrak{A}$.

Proof Immediately from Claim 1.

The demand that Bueno dismisses is recovered by substituting '=' for ' $\cong$ ' in Definition 14 and Claim 3. ${ }^{19}$ Note that, besides allowing for approximations more general than those that can be described by partial structures, Definition 14 additionally avoids the need for partial theories.

\subsection{Pragmatic empirical adequacy}

Bueno's second generalization (Bueno 1997, §5) starts from the ideas that the structures of data are often changed in multiple steps into structures of theories (Suppes 1962), and that theories are not compared directly to data, but more refined structures (Bogen and Woodward 1988). To capture both points, Bueno (1997, pp. 600-601) gives an informal description of a hierarchy of partial structures (cf. French and Ladyman 1999, p. 113). Since he makes a distinction between partial structures with finite and with infinite domains, this can be captured in the following two more explicit definitions.

Definition 16 A hierarchy of finite partial structures is a finite sequence $\left\langle\tilde{\mathfrak{B}}_{l}\right\rangle_{1 \leq l \leq m}$ of relational partial structures with finite domains for the $\lambda_{i}$-place relation symbols $\left\{R_{i}\right\}_{i \in I},{ }^{20}$ such that (i) $\tilde{\mathfrak{B}}_{m}$ corresponds to a structure, and (ii) for all $1 \leq l<m$ and $i \in I, \operatorname{card}\left(R_{i}^{\tilde{\mathfrak{B}}_{l}, \circ}\right)>\operatorname{card}\left(R_{i}^{\tilde{\mathfrak{B}}_{l+1}, \circ}\right)$ and $\operatorname{card}\left(B_{l}\right) \leq \operatorname{card}\left(B_{l+1}\right) .^{21}$

Definition 17 A hierarchy of infinite partial structures is a finite sequence $\left\langle\tilde{\mathfrak{B}}_{l}\right\rangle_{1 \leq l \leq m}$ of relational partial structures with infinite domains for the $\lambda_{i}$-place relation symbols $\left\{R_{i}\right\}_{i \in I}$, such that (i) $\tilde{\mathfrak{B}}_{m}$ corresponds to a structure and (ii) for all $1 \leq l<m$ and $i \in I, R_{i}^{\tilde{\mathfrak{B}}_{l}, \circ} \supset R_{i}^{\tilde{\mathfrak{B}}_{l+1}, \circ}$.

A hierarchy of partial structures is either a hierarchy of finite or a hierarchy of infinite partial structures.

Bueno (1997, p. 601) further defines a reduced partial structure $\tilde{\mathfrak{B}}$ of a partial structure $\tilde{\mathfrak{C}}$ to be a partial substructure of $\tilde{\mathfrak{C}}$ such that every element of $\tilde{\mathfrak{B}}$ 's domain has been measured at some point with a specific measurement result. Finally, Bueno (1997, pp. 601,607$)$ seems to define the partial models of the phenomena to be a hierarchy of partial structures (a) whose lowest partial structure represents the data, (b) whose

\footnotetext{
19 The set theoretic result used in Sect. 4.1 (Hodges 1993, ex. 1.2.4b) holds only for single embeddings and can therefore not be used to counter Bueno's objection.

20 That all structures are for the same relation symbols is only implicitly suggested by (my paraphrase of) Bueno's informal description of the partial models of the phenomena below.

${ }^{21}$ Given that all structures in the domain are for the same relation symbols and thus specifically for relation symbols with the same respective number of arguments, the cardinality condition on the domains is implicit in Bueno's claim that from $\operatorname{card}\left(R_{i}^{\tilde{\mathfrak{B}}_{l}, \circ}\right)>\operatorname{card}\left(R_{i}^{\tilde{\mathfrak{B}}_{l+1}, \circ}\right)$, it follows that $\operatorname{card}\left(R_{i}^{\tilde{\mathfrak{B}}_{l},+}\right)<\operatorname{card}\left(R_{i}^{\tilde{\mathfrak{B}}_{l+1},+}\right)$ $\operatorname{or} \operatorname{card}\left(R_{i}^{\tilde{\mathfrak{B}}_{l,-}}\right)<\operatorname{card}\left(R_{i}^{\tilde{\mathfrak{B}}_{l+1},-}\right)$.
} 
highest partial structure represents the appearances, and (c) where there is a cardinal number $w$ such that the partial structure at each level has a reduced partial structure with a domain of cardinality $w .^{22}$ By Definition 11 , there is for every relational partial structure $\tilde{\mathfrak{B}}$ and set $C \subseteq B$ a partial substructure $\tilde{\mathfrak{C}} \subseteq \tilde{\mathfrak{B}}$ with $\operatorname{dom}(\tilde{\mathfrak{C}})=C$. Thus condition (c) simply demands that for each $l, 1 \leq l \leq m$, at least one element of domain $A_{l}$ has been measured.

Bueno (1997, p. 601, my notation) claims that partial models of the phenomena formalize the idea that

at each level, partial relations $R$, which were not yet defined at a lower level (whose elements thus belong to $R^{\circ}$ ) come to be defined: either taking their elements as belonging to the domain of $R$ (i.e. $R^{+}$) or belonging to the complement of $R$ (that is, $R^{-}$). The partial relations are extended until one obtains normal (total) structures. These are then to be compared to scientific theories in testing them.

This description is rather misleading because it incorrectly suggests that each new level in the hierarchy introduces new relations. What is probably meant is that at each level $\tilde{\mathfrak{B}}_{l}$ and for each relation symbol $R_{i}$, tuples of members of the domain that were borderline cases at the lower level $\tilde{\mathfrak{B}}_{l-1}$ (and thus belong to $R_{i}^{\tilde{\mathfrak{B}}_{l-1}, \circ}$ ) come to be included either in the positive extension of $R_{i}$ (i.e., $R_{i}^{\tilde{\mathfrak{B}}_{l},+}$ ) or in the negative extension of $R_{i}$ (i.e., $\left.R_{i}^{\tilde{\mathfrak{B}}_{l},-}\right)^{23}$

The definition of a hierarchy of partial structures has a number of quirks that become problems in the definition of the partial models of the phenomena. One is that a partial structure $\tilde{\mathfrak{B}}_{l}$ with $R_{i}^{\tilde{\mathfrak{B}}_{l}, \circ}=\varnothing$ for some, but not all $i \in I$ cannot occur in a hierarchy. For condition (ii) demands that $\operatorname{card}\left(R_{i}^{\tilde{\mathfrak{B}}_{l}, \circ}\right)>\operatorname{card}\left(R_{i}^{\tilde{\mathfrak{B}}_{l+1}, \circ}\right)$ or $R_{i}^{\tilde{\mathfrak{B}}_{l}, \circ} \supset R_{i}^{\tilde{\mathfrak{B}}_{l+1}, \circ}$ for all $i \in I$. Thus, if $R_{i}^{\tilde{\mathfrak{B}}_{l}, \circ}=\varnothing$ for some $i \in I$, condition (ii) cannot be fulfilled by $R_{i}^{\tilde{\mathfrak{B}}_{l+1} \text {, }}$, so that $\mathfrak{B}_{l}$ must be the highest level of the hierarchy. And because the highest level must correspond to a structure, $R_{i}^{\tilde{\mathfrak{B}}_{l}, \circ}=\varnothing$ for all $i \in I$. This problem can easily be solved by slightly modifying condition (ii), demanding only $\operatorname{card}\left(R_{i}^{\tilde{\mathfrak{B}}_{l, \circ}}\right) \geq \operatorname{card}\left(R_{i}^{\tilde{\mathfrak{B}}_{l+1}, \circ}\right)$ in Definition 16 and $R_{i}^{\tilde{\mathfrak{B}}_{l}, \circ} \supseteq R_{i}^{\tilde{\mathfrak{B}}_{l+1}, \circ}$ in Definition 17 for all $i \in I$. To arrive at an asymmetric relation, one could further demand that the respective converse relation does not hold for all $i \in I$.

A bigger problem is that the demands on the relations are radically different for hierarchies of finite and infinite domains. Infinite structures form a hierarchy if from one level to the next, some borderline cases of each relation symbol become members of its positive or negative extension, but not vice versa. The relations of finite structures, on the other hand, only have to have a decreasing number of borderline cases, no matter which ones they are. This means that the concept of a hierarchy of infinite partial structures does not constitute a limit of the concept of a hierarchy of finite

22 Conditions (a) and (b) are inferred from Bueno's informal discussion.

23 Bueno (email from 5 January, 2011) has confirmed that this is an accurate paraphrase. 
partial structures: If $\tilde{\mathfrak{B}}_{l}=\left\langle B, R_{1}^{\tilde{\mathfrak{B}}_{l}}\right\rangle$ with $R_{1}^{\tilde{\mathfrak{B}}_{l}, \circ}=\{a, b\}$, and $\tilde{\mathfrak{B}}_{l+1}=\left\langle B, R_{1}^{\tilde{\mathfrak{B}}_{l+1}}\right\rangle$ with $R_{1}^{\tilde{\mathfrak{B}}_{l+1}, \circ}=\{c\}$, then $\operatorname{card}\left(R_{i}^{\tilde{\mathfrak{B}}_{l}, \circ}\right)>\operatorname{card}\left(R_{i}^{\tilde{\mathfrak{B}}_{l+1}, \circ}\right)$, but $R_{i}^{\tilde{\mathfrak{B}}_{l}, \circ} \not \supset R_{i}^{\tilde{\mathfrak{B}}_{l+1}, \circ}$. Thus these two structures can be elements of hierarchies of finite partial structures with arbitrarily big finite domains $B$, but cannot be elements of a hierarchy of infinite partial structures. Note also that for infinite domains, the elements in $R_{i}^{\tilde{\mathfrak{B}}_{l}, \circ}$ for any $i \in I, 1 \leq l \leq m$ have to be in every $R_{i}^{\tilde{\mathfrak{B}}_{k}, \circ}, k \leq l$, so that, since $R_{i}^{\tilde{\mathfrak{B}}_{l}, \circ} \neq 0$ for all $i \in I, 1 \leq l<m$, partial structures at all levels except the last one have to have some elements in common. In contradistinction, the domains of the finite structures can change completely from one level to the next.

This radical difference between hierarchies of finite and infinite structures has a number of odd implications. First and foremost, there is no obvious reason for the change in condition (ii). If the subset-relation is justified for infinite domains, its lack of justification for finite domains first has to be shown. Second, it is not clear how to define a hierarchy of partial structures some of which have finite, and some of which have infinite domains. Third, any concept relying on the concept of a hierarchy of partial structures will also have very different properties for finite and infinite domains. This, though, renders typical scientific methods unusable, for example the use of limiting cases, where a very big but finite domain is used as approximation of an infinite domain. Results for finite domains thus will typically have little bearing on results for infinite domains, and vice versa. ${ }^{24}$

The biggest problem of the concept of a hierarchy of partials structures, however, is starkly illustrated by Bueno's own informal description (quoted above) of each level in the hierarchy as "extending" the relations of the partial structure from the previous level. In the rest of his article, Bueno (e.g., 1997, pp. 592-593) uses the concept of one relation "extending" another only to state that the relations $\left\{R_{i}^{\mathfrak{B}}\right\}_{i \in I}$ in a $\tilde{\mathfrak{B}}_{l}$-normal structure $\mathfrak{B}$ extend the relations $\left\{R_{i}^{\tilde{\mathfrak{B}}_{l}}\right\}_{i \in I}$. This entails that to $\mathfrak{B}$ there corresponds a partial structure $\tilde{\mathfrak{B}}$ so that for all $i \in I, R_{i}^{\tilde{\mathfrak{B}}_{l},+} \subseteq R_{i}^{\tilde{\mathfrak{B}},+}$ and $R_{i}^{\tilde{\mathfrak{B}}_{l},-} \subseteq R_{i}^{\tilde{\mathfrak{B}},-}$. The natural generalization of this use of 'extension' is that the same subset condition also holds between all relations of any two partial structures where the relations of the one extend the relations of the other. ${ }^{25}$ But understood in this way, Bueno's informal description is incompatible with his definition of hierarchies of infinite and of finite partial structures. In hierarchies of infinite partial structures, a member of the positive extension $R_{i}^{\tilde{\mathfrak{B}}_{l},+}$ may be in the negative extension $R_{i}^{\tilde{\mathfrak{B}}_{l+1},-}$

\footnotetext{
24 It is straightforward to adapt the definition of a hierarchy of infinite partial structures to finite domains. To adapt the definition of a hierarchy of finite partial structures to infinite domains, one could assign to each $k$-ary relation $R_{i}^{\tilde{\mathfrak{B}}_{l}}$ a normalized measure $\mu_{l, i}$ over $A_{l}^{k}$ and demand that ii) for all $1 \leq l<m$ and $i \in I, \mu\left(R_{i}^{\tilde{\mathfrak{B}}_{l}, \circ}\right)>\mu\left(R_{i}^{\tilde{\mathfrak{B}}_{l+1}, \circ}\right)$ and $\operatorname{card}\left(A_{l}\right) \geq \operatorname{card}\left(A_{l+1}\right)$. The definition of a hierarchy of finite partial structures would then be recovered by the definition $\mu\left(R_{i}^{\tilde{\mathfrak{B}}_{l}, \circ}\right)=\operatorname{card}\left(R_{i}^{\tilde{\mathfrak{A}}_{l}, \circ}\right) / \operatorname{card}\left(A_{l}^{k}\right)$. This solution, however, would go beyond the partial structure formalism and would require a justification of each measure $\mu_{l, i}$.

25 This is what Bueno and de Souza (1996, definition 5) call an 'expansion' of one partial structure by another.
} 
and vice versa. In hierarchies of finite partial structures, any instance, non-instance, or borderline case can become an instance, non-instance, or borderline case on the next level of the hierarchy, as long as the number of borderline cases overall decreases. Hence at each level, any information one has about the domain can be negated on the next level. It is thus a stretch to say that the hierarchy is "built in such a way that, at each level, there is a gain of information regarding the phenomena being modeled" (Bueno 1997, p. 603), because then one would convey information by saying: "They are certainly scoundrels, though they may not be". ${ }^{26}$

This problem is severe enough that the relation of the highest level of a pragmatic empirical hierarchy to the rest of the hierarchy is almost trivial:

Claim 4 If $\left\langle\tilde{\mathfrak{B}}_{l}\right\rangle_{1 \leq l \leq m}$ is a hierarchy of infinite partial structures for $\left\{R_{i}\right\}_{i \in I}$, then $\left\langle\tilde{\mathfrak{B}}_{l}, \tilde{\mathfrak{C}}\right\rangle_{1 \leq l<m}$ is also a hierarchy of infinite partial structures for any infinite partial structure $\tilde{\mathfrak{C}}$ for $\left\{R_{i}\right\}_{i \in I}$ that corresponds to a structure. If $\left\langle\tilde{\mathfrak{B}}_{l}\right\rangle_{1 \leq l \leq m}$ is a hierarchy of finite partial structures for $\left\{R_{i}\right\}_{i \in I}$, then $\left\langle\tilde{\mathfrak{B}}_{l}, \tilde{\mathfrak{C}}_{\rangle_{1 \leq l<m}}\right.$ is also a hierarchy of finite partial structures for any finite partial structure $\tilde{\mathfrak{C}}$ for $\left\{R_{i}\right\}_{i \in I}$ with $\operatorname{card}\left(B_{m-1}\right) \leq$ card $(\operatorname{dom}(\tilde{\mathfrak{C}}))$ that corresponds to a structure. (This holds whether the definitions use 'つ’, ' $\supseteq$ ', '> , or ' $\geq$ ' in condition (ii).)

Proof If condition (ii) is formulated with ' $\supset$ ', then $R_{i}^{\tilde{\mathfrak{B}}_{m-1}, \circ} \neq \varnothing$ for all $i \in I$. Since $R_{i}^{\tilde{\mathfrak{C}}, \circ}=\varnothing$ for all $i \in I$ and the empty set is a proper subset of every nonempty set, condition (ii) is fulfilled. If condition (ii) is formulated with ' $>$ ', then $\operatorname{card}\left(R_{i}^{\tilde{\mathfrak{B}}_{m-1}, \circ}\right)>0=\operatorname{card}\left(R_{i}^{\tilde{\mathfrak{C}}, \circ}\right)$ for all $i \in I$. If condition (ii) is formulated with ' $\supseteq$ ' or ' $\geq$ ', the proof is immediate.

Therefore any level of a hierarchy of partial structures below the highest one only restricts the relation symbols of the partial structure at the highest level (and in the finite case also the minimal size of the domain). ${ }^{27}$ This almost trivial restriction of the highest level of the hierarchy of partial structures by the lower levels is not meaningfully strengthened in Bueno's definition of the partial models of the phenomena, for the only additional restriction on the structure at the highest level of the hierarchy is that at least one object in its domain must have been measured. Which element is measured, however, is independent from the lower levels.

Bueno (1997, p. 602, my notation) goes on to define that a theory

is empirically adequate if it is pragmatically true in the (partial) empirical substructure $\tilde{\mathfrak{E}}$ according to a structure $\mathfrak{B}$, where $\mathfrak{B}$ is the last level of the hierarchy of models of phenomena (being thus a total structure).

A sentence is "pragmatically true in $\tilde{\mathfrak{E}}$ according to $\mathfrak{B}$ " if and only if it is true in $\mathfrak{B}$ and $\mathfrak{B}$ is $\tilde{\mathfrak{E}}$-normal (Bueno 1997, p. 592, my notation). Technically, $\mathfrak{B}$ is the total structure

\footnotetext{
26 In some contexts, of course, this sentence can convey information by way of conversational implicature (e. g., Hammett 1928, pp. 115-120).

27 These criticisms also apply to the applications of the hierarchies by, for instance, Bueno (1999, §3.1), French and Ladyman (1999, pp. 112-114) Bueno et al. (2002, §2), da Costa and French (2003, pp. 68-74), Bueno and Colyvan (2011, §5.3, n. 25), and French (2014, p. 128).
} 
that corresponds to the last level $\tilde{\mathfrak{B}}$ of the hierarchy of models of phenomena, since each level in such a hierarchy is a partial structure.

Bueno's definition of what I will call 'pragmatic empirical adequacy' requires some unpacking, since it defines the pragmatic empirical adequacy of a sentence (and thus, via Tarski semantics, of a class or a family of structures), not the pragmatic empirical adequacy of a family of partial structures. But Bueno's definition presumes that theories have partial empirical substructures, hence it presumes that theories are at the same time given as families of partial structures. Luckily, this puzzle is easily solved by relying on the suggestion for arriving at partial structures of theories that was developed in Sect. 4.1. The suggestion provides, for a theory given by a sentence $\vartheta$ and thus by a family of structures $\left\{\mathfrak{T}_{n}\right\}_{n \in N}$, a corresponding family of partial structures $\left\{\tilde{\mathfrak{T}}_{m}\right\}_{m \in M}$. Hence one can state Bueno's definition as follows: A theory $\vartheta$ with the corresponding partial theory $\left\langle\left\{\tilde{\mathfrak{T}}_{m}\right\}_{m \in M},\left\{\tilde{\mathbf{E}}_{m}\right\}_{m \in M}\right\rangle$ is pragmatically empirically adequate iff there is an $m \in M$ and an $\tilde{\mathfrak{E}} \in \tilde{\mathbf{E}}_{m}$ such that $\vartheta$ is pragmatically true in $\tilde{\mathfrak{E}}$ according to $\mathfrak{B}$, where $\mathfrak{B}$ is the last level of the hierarchy of models of phenomena. Given the definition of 'pragmatically true in $\tilde{\mathfrak{E}}$ according to $\mathfrak{B}$ ', this is means that $\vartheta$ is pragmatically empirically adequate iff $\vartheta$ is true in $\mathfrak{B}$, where $\mathfrak{B}$ is the last level of the hierarchy of models of phenomena and is $\tilde{\mathfrak{E}}$-normal for some $m \in M$ and $\tilde{\mathfrak{E}} \in \tilde{\mathbf{E}}_{m}$. If the theory is expressed as a family $\left\langle\mathfrak{T}_{n}, \mathbf{E}_{n}\right\rangle$ of structures, Bueno's definition entails that $\left\langle\mathfrak{T}_{n}, \mathbf{E}_{n}\right\rangle$ is pragmatically empirically adequate iff there is an $n \in N$, an $m \in M$, and an $\tilde{\mathfrak{E}} \in \tilde{\mathbf{E}}_{m}$ such that $\mathfrak{T}_{n}$ is $\tilde{\mathfrak{E}}$-normal and the last level of the hierarchy of models of phenomena.

This definition has a number of peculiar features that cast its adequacy into doubt. For one, it defines pragmatic empirical adequacy relative to a single hierarchy of partial structures, with a single structure on its highest level that represents the appearances. Hence the definition presumes, contrary to van Fraassen, that there is only one appearance. Second, since $\mathfrak{T}_{n}$ is $\tilde{\mathfrak{E}}$-normal, $\operatorname{dom}\left(\mathfrak{T}_{n}\right)=E$, so that all the objects that occur in the theory also have to occur in its partial empirical substructure, which thereby is not a proper substructure. Third, every element of the approximation set corresponding to $\tilde{\mathfrak{E}}_{n}$ is $\tilde{\mathfrak{E}}_{n}$-normal, so that Bueno's definition entails the following: $\left\langle\mathfrak{T}_{n}, \mathbf{E}_{n}\right\rangle$ is pragmatically empirically adequate iff there is an $n \in N$ such that $\mathfrak{T}_{n}$ is the last level of the hierarchy of models of phenomena. In other words, a theory is pragmatically empirically adequate if and only if it is true in the highest level of the partial models of the phenomena, that is, in the single appearance. ${ }^{28}$ Thus empirical adequacy is the same as truth in the structure of the appearance, which trivializes van Fraassen's idea of substituting empirical adequacy for truth as the goal of science. Fourth, there is only the almost trivial relation between the appearances at the highest level and the data at the lowest level of the models of the phenomena. Claim 4 shows that the last level is almost independent from the lower levels, so that, if the data are meant to determine the appearances, the notion of pragmatic empirical adequacy is almost trivial. If, on the other hand, the appearances are determined on their own, then pragmatic empirical adequacy simply presumes that the appearance is given by a structure, not a partial structure, and thus does not differ in this respect from empirical adequacy. A corollary of these four points is that pragmatic empirical adequacy clearly does not properly gen-

$\overline{28 \text { This means that the notion of }}$ pragmatic truth plays no role in pragmatic empirical adequacy. 
eralize empirical adequacy. The effects of these modifications of empirical adequacy on Suárez's criticism are discussed in "Appendix C.2".

The fifth peculiarity of the definition is that it contradicts the presupposition of Bueno's claim that "there is no problem with the fact that $\tilde{\mathfrak{E}}$ and $\mathfrak{B}$ have distinct domains" (Bueno 1997, p. 603, my notation). There is indeed no problem, but only because $\tilde{\mathfrak{E}}$ and $\mathfrak{B}=\mathfrak{T}_{n}$ have the same domain, since $\mathfrak{B}$ is $\tilde{\mathfrak{E}}$-normal. Oddly, Bueno (1997, p. 603, n. 16) seems to acknowledge this in a footnote, in which he states that "one of the conditions for a total structure to be employed as one that extends the relations of a partial structure, is that both of them have the same domain. Such a condition obviously could not hold here." ${ }^{29}$ This identity of the domain of the partial appearance and the partial empirical substructure should be fatal for the definition according to Bueno's own standards, since he has dismissed another suggestion for generalizing empirical adequacy (discussed in connection with Claim 3) on exactly these grounds.

Finally, the definition is in tension with Bueno's informal description. Bueno (1997, p. 603) states:

The basic point of [the definition] consists in the fact that, intuitively, a theory is empirically adequate if that part of it which is concerned with the observable phenomena (its empirical substructures) can be extended to a total structure that represents the information provided by the observational side of 'experience' (the last level in the hierarchy of partial models of phenomena).

If $\left\{\tilde{\mathfrak{T}}_{m}\right\}_{m \in M}$ is given via approximation sets, and thus the partial empirical substructures $\tilde{\mathfrak{E}}$ are given via approximation sets as well, the $\tilde{\mathfrak{E}}$-normal structures are just empirical substructures of $\left\langle\mathfrak{T}_{n}, \mathbf{E}_{n}\right\rangle$. Further, the highest level of the models of the phenomena corresponds to a structure $\mathfrak{A}$. If a structure is represented by another through an isomorphism between the two, Bueno's informal description then states that a theory is pragmatically empirically adequate if and only if one of its empirical substructures is isomorphic to the appearance $\mathfrak{A}$. In Bueno's informal description, pragmatic empirical adequacy thus differs from empirical adequacy only in that there can be only one appearance, and there must also be an almost unrelated data structure. But this is not the case for Bueno's more formal definition.

The first part of this discussion (Lutz 2014b, def. 14, 15, 17) contains a generalization of empirical adequacy that avoids all of the above criticisms. It provides a substitute for Bueno's notion of the models of the phenomena:

Definition 18 A restricted hierarchy of approximate appearances $\left\langle\left\{\mathbf{A}_{l}\right\}_{l \in L}, C\right\rangle$ contains an indexed set $\left\{\mathbf{A}_{l}\right\}_{l \in L}$ of approximate appearances with an ordered index set $L$ and a set $C$ containing for any $\mathbf{A}_{l}, \mathbf{A}_{m}, l, m \in L, l \leq m$ exactly one bijection $b: \mathbf{A}_{l} \longrightarrow \mathbf{A}_{m}$ such that for all $\mathbf{Q} \in \mathbf{A}_{l}, b(\mathbf{Q}) \subseteq \mathbf{Q}$.

Additionally, for any three bijections $b, c, d \in C$ with $b: \mathbf{A}_{l} \longrightarrow \mathbf{A}_{m}, c: \mathbf{A}_{m} \longrightarrow$ $\mathbf{A}_{n}$, and $d: \mathbf{A}_{l} \longrightarrow \mathbf{A}_{n}, d=c \circ b$.

\footnotetext{
${ }^{29} \mathrm{He}$ adds that "the condition can be easily changed for a weaker one, still preserving the main features of the concept of pragmatic truth. I have pursued this line of argument elsewhere [...]." The referenced article (Bueno and de Souza 1996), however, gives no indication on how to avoid the identity of the domains of $\tilde{\mathfrak{E}}$ and $\mathfrak{B}$, as it does not discuss possible relations between partial structures with different domains.
} 
As explained in the first part of this discussion, this definition leads to a real increase of information from one point of the hierarchy to the next, allows more than one appearance, and is not restricted to hierarchies of finitely many levels; ${ }^{30}$ with the help of the set $C$, it also determines for any appearance at any level in the hierarchy a unique line of ancestors and descendants and thus, specifically, which appearances from different levels describe the same phenomenon.

One can define which appearances are possible given the knowledge one has about them at some point (cf. Lutz 2014b, def. 11):

Definition 19 Given approximate appearances $\mathbf{A}, \mathbf{A}^{\prime}$ are approximately possible appearances if and only if $\mathbf{A}^{\prime}=\{e(\mathbf{Q}): \mathbf{Q} \in \mathbf{A}\}$, where $e$ is any function from $\mathbf{A}$ to $\bigcup \mathbf{A}$ with $e(\mathbf{Q}) \in \mathbf{Q}$.

Thus for every approximation set in the approximate appearances, any of its members can be a member of the appearances.

These definitions lead to the following result (Lutz 2014b, Claim 7):

Claim $5\left\langle\mathfrak{T}_{n}, \mathbf{E}_{n}\right\rangle$ is approximately empirically adequate at all points of all restricted hierarchies of approximate appearances with the initial sequence $\left\langle\mathbf{A}_{l}\right\rangle_{l \leq m}$ if and only if $\left\langle\mathfrak{T}_{n}, \mathbf{E}_{n}\right\rangle$ is empirically adequate for all appearances that are approximately possible given $\mathbf{A}_{l}$.

By Claim 5, the growth of information can be represented by the restricted hierarchy of approximate appearances, and at each point of the hierarchy, it is clear which theories are approximately empirically adequate. Thus, while Bueno's generalizations based on partial structures are inadequate, his motivations provide reasons for adopting the generalizations suggested in the first part of this discussion.

\section{Conclusion}

Partial structures and van Fraassen's notion of empirical adequacy do not go well together: Empirical adequacy has been neither successfully expressed nor successfully generalized in terms of partial structures. Partial structures even face problems in their initial intended domain of application: They are inadequate for expressing approximations of functions and constants.

The notion of an approximation set generalizes the notion of a partial structure to allow for approximations of functions and constants, and allows for the notions of partial isomorphism and partial homomorphism without the use of partial structures. These results show that approximation sets can be used to formalize all theories, models, and appearances that can be formalized in partial structures, and can in fact formalize more. That approximation sets do not rely on any new technical notions allows for a simple transfer of known results from model theory. Furthermore, approximation sets are the basis of the notion of approximate empirical adequacy and of a restricted hierarchy of approximate empirical appearances, which capture the basic intuitions behind Bueno's notions of partial and pragmatic empirical adequacy without succumbing to their problems.

30 Indeed, the index set $L$ can be any well-ordered set and thus, for example, uncountably infinite. 
Together with the results of the first part of this discussion, these results show the adequacy and flexibility of approximation sets, approximate empirical adequacy, and hierarchies of approximate empirical appearances.

Acknowledgements An early version of this paper has been presented at Herman Philipse's Dutch Research Seminar in Analytic Philosophy at the University of Utrecht. Many thanks to the participants and Anthony Booth, Thomas Müller, and especially Otávio Bueno for helpful discussions. Thanks also to a reviewer of this journal, who provided detailed and very helpful feedback on the technical aspects of this paper.

Open Access This article is distributed under the terms of the Creative Commons Attribution 4.0 International License (http://creativecommons.org/licenses/by/4.0/), which permits unrestricted use, distribution, and reproduction in any medium, provided you give appropriate credit to the original author(s) and the source, provide a link to the Creative Commons license, and indicate if changes were made.

\section{Appendix A: Partial isomorphisms and partial homomorphisms in terms of approximation sets}

Claim 6 Let $\mathbf{B}$ and $\mathbf{C}$ be the approximation sets corresponding to partial structures $\tilde{\mathfrak{B}}$ and $\tilde{\mathfrak{C}}$ with $\tilde{\Pi}=\varnothing$. Then $f$ is a partial isomorphism between $\tilde{\mathfrak{B}}$ and $\tilde{\mathfrak{C}}$ if and only if for every $\mathfrak{B} \in \mathbf{B}$ there is a $\mathfrak{C} \in \mathbf{C}$ and for every $\mathfrak{C} \in \mathbf{C}$ there is a $\mathfrak{B} \in \mathbf{B}$ such that $f$ is an isomorphism between $\mathfrak{B}$ and $\mathfrak{C}$.

Proof ' $\Rightarrow$ ': Let $f: B \longrightarrow C$ be a partial isomorphism between $\tilde{\mathfrak{B}}$ and $\tilde{\mathfrak{C}}$ and let $\mathfrak{B}$ be in $\mathbf{B}$. By Claim $1, \mathfrak{B}$ is $\tilde{\mathfrak{B}}$-normal for $\varnothing$. Since $f$ is a bijection, it is an isomorphism from $\mathfrak{B}$ to $\mathfrak{C}$ with $R_{i}^{\mathfrak{C}}=f\left(R_{i}^{\mathfrak{B}}\right)$ for each $i \in I, F_{j}^{\mathfrak{C}} x_{1} \ldots x_{\rho_{j}}=$ $f\left(F_{j}^{\mathfrak{B}} f^{-1}\left(x_{1}\right) \ldots f^{-1}\left(x_{\rho_{j}}\right)\right)$ for each $x_{1}, \ldots, x_{\rho_{j}} \in C, j \in J$, and $c_{k}^{\mathfrak{C}}=f\left(c_{k}^{\mathfrak{B}}\right)$

for each $k \in K$. Furthermore, $\mathfrak{C}$ is $\tilde{\mathfrak{C}}^{-}$-normal, because first, $R_{i}^{\tilde{\mathfrak{C}},+}=f\left(R_{i}^{\tilde{\mathfrak{B}},+}\right) \subseteq$ $f\left(R_{i}^{\mathfrak{B}}\right)=R_{i}^{\mathfrak{C}} \subseteq f\left(R_{i}^{\tilde{\mathfrak{B}},-}\right)=R_{i}^{\tilde{\mathfrak{C}},-}$. Second, $W_{\tilde{\mathfrak{B}}, j}=f\left(W_{\tilde{\mathfrak{B}}, j}\right)$ and hence $F_{j}^{\mathfrak{C}} x_{1} \ldots x_{\rho_{j}}=f\left(F_{j}^{\mathfrak{B}} f^{-1}\left(x_{1}\right) \ldots f^{-1}\left(x_{\rho_{j}}\right)\right)=f\left(F_{j}^{\tilde{\mathfrak{B}}} f^{-1}\left(x_{1}\right) \ldots f^{-1}\left(x_{\rho_{j}}\right)\right)=$ $F_{j}^{\tilde{\mathfrak{C}}} x_{1} \ldots x_{\rho_{j}}$ for all $x_{1}, \ldots, x_{\rho_{j}} \in W_{\tilde{\mathfrak{C}}}$. Third, $c_{k}^{\mathfrak{C}}=f\left(c_{k}^{\mathfrak{B}}\right)=f\left(c_{k}^{\tilde{\mathfrak{B}}}\right)=c_{k}^{\tilde{\mathfrak{B}}}$. By Claim $1, \mathfrak{C} \in \mathbf{C}$. By the same reasoning, if $\mathfrak{C} \in \mathbf{C}$, there is a $\mathfrak{B} \in \mathbf{B}$ such that $f^{-1}$ and thus $f$ is an isomorphism between $\mathfrak{B}$ and $\mathfrak{C} .^{31}$

' $\Leftarrow$ ': Assume that $f: B \longrightarrow C$ is a bijection but not a partial isomorphism between $\tilde{\mathfrak{B}}$ and $\tilde{\mathfrak{C}}$. Then there are an $i \in I$ and some $x_{1}, \ldots, x_{\lambda_{i}} \in B$ such that (i) $R_{i}^{\mathfrak{B},+} x_{1} \ldots x_{\lambda_{i}}$ and not $R_{i}^{\mathfrak{C},+} f\left(x_{1}\right) \ldots f\left(x_{\lambda_{i}}\right)$ or (ii) not $R_{i}^{\mathfrak{B},+} x_{1} \ldots x_{\lambda_{i}}$ and $R_{i}^{\mathfrak{C},+} f\left(x_{1}\right) \ldots f\left(x_{\lambda_{i}}\right)$ or (iii) $R_{i}^{\mathfrak{B},-} x_{1} \ldots x_{\lambda_{i}}$ and not $R_{i}^{\mathfrak{C},-} f\left(x_{1}\right) \ldots f\left(x_{\lambda_{i}}\right)$ or (iv) not $R_{i}^{\mathfrak{B},-} x_{1} \ldots x_{\lambda_{i}}$ and $R_{i}^{\mathfrak{C},-} f\left(x_{1}\right) \ldots f\left(x_{\lambda_{i}}\right)$, or there is a $j \in J$ such that for some $x_{1}, \ldots, x_{\rho_{j}} \in B,(\mathrm{v}) W_{\tilde{\mathfrak{B}}, j} x_{1} \ldots x_{\rho_{j}}$ and not $W_{\tilde{\mathfrak{C}}, j} f\left(x_{1}\right) \ldots f\left(x_{\rho_{j}}\right)$ or (vi) not $W_{\tilde{\mathfrak{B}}, j} x_{1} \ldots x_{\rho_{j}}$ and $W_{\tilde{\mathfrak{C}}, j} f\left(x_{1}\right) \ldots f\left(x_{\rho_{j}}\right)$ or (vii) $W_{\tilde{\mathfrak{B}}, j} x_{1} \ldots x_{\rho_{j}}$ and $f\left(F_{j}^{\tilde{\mathfrak{B}}} x_{1} \ldots x_{\rho_{j}}\right) \neq F_{j}^{\tilde{\mathfrak{C}}} f\left(x_{1}\right) \ldots f x_{\rho_{j}}$, or (viii) there is a $k \in K$ such that $f c_{k}^{\tilde{\mathfrak{B}}} \neq c_{k}^{\tilde{\mathfrak{C}}}$. It is to be shown that $(*)$ there is a $\mathfrak{B} \in \mathbf{B}$ for which there is no $\mathfrak{C} \in \mathbf{C}$ or there is a $\mathfrak{C} \in \mathbf{C}$ for which there is no $\mathfrak{B} \in \mathbf{B}$ such that $f$ is an isomorphism between $\mathfrak{B}$ and $\mathfrak{C}$.

\footnotetext{
31 This half of the proof generalizes the rough proof given by Bueno (2000, pp. 279-280).
} 
If (i) holds for some $i \in I$ and $x_{1}, \ldots, x_{\lambda_{i}}$, then choose a $\tilde{\mathfrak{B}}$-normal structure with $R_{i}^{\mathfrak{B}} x_{1} \ldots x_{\lambda_{i}}$, if (ii) holds for some $i \in I$ and $x_{1}, \ldots, x_{\lambda_{i}}$, then choose a $\tilde{\mathfrak{C}}$-normal structure with $R_{i}^{\mathfrak{C}} f\left(x_{1}\right) \ldots f\left(x_{\lambda_{i}}\right)$, and analogously for (iii) and (iv). Then, if $f$ is an isomorphism between $\mathfrak{B}$ and $\mathfrak{C}$, in case (i) $\mathfrak{C}$ is not $\tilde{\mathfrak{C}}$-normal because $R_{i}^{\mathfrak{C},+} f\left(x_{1}\right) \ldots f\left(x_{\lambda_{i}}\right)$ does not hold, in case (ii) $\mathfrak{B}$ is not $\tilde{\mathfrak{B}}$-normal because $R_{i}^{\mathfrak{B},+} x_{1} \ldots x_{\lambda_{i}}$ does not hold, and analogously for (iii) and (iv). If (v) holds for some $j \in J$ and $x_{1}, \ldots, x_{\rho_{j}} \in B$, choose $F_{j}^{\mathfrak{C}} f\left(x_{1}\right), \ldots, f\left(x_{\rho_{j}}\right) \neq f\left(F_{j}^{\tilde{\mathfrak{B}}} x_{1} \ldots x_{\rho_{j}}\right)$, and analogously for (vi). Then, if $f$ is an isomorphism between $\mathfrak{B}$ and $\mathfrak{C}$, in case (v) $\mathfrak{B}$ is not $\tilde{\mathfrak{B}}$-normal because $F_{j}^{\mathfrak{B}} x_{1} \ldots x_{\rho_{j}}=f^{-1}\left(F_{j}^{\mathfrak{C}} f\left(x_{1}\right) \ldots f\left(x_{\rho_{j}}\right)\right) \neq F_{j}^{\tilde{\mathfrak{B}}} x_{1} \ldots x_{\rho_{j}}$, and analogously for (vii). For any $\tilde{\mathfrak{B}}$-normal structure $\mathfrak{B}$ and any $\tilde{\mathfrak{C}}$-normal structure $\mathfrak{C}$, if (vii) holds, $F_{j}^{\mathfrak{B}} x_{1}, \ldots, x_{\rho_{j}}=F_{j}^{\tilde{\mathfrak{B}}} x_{1} \ldots x_{\rho_{j}} \neq F_{j}^{\tilde{\mathfrak{C}}} x_{1}, \ldots, x_{\rho_{j}}=F_{j}^{\mathfrak{C}} x_{1}, \ldots, x_{\rho_{j}}$ and if (viii) holds, $c_{k}^{\mathfrak{B}}=c_{k}^{\tilde{\mathfrak{B}}} \neq c_{k}^{\tilde{\mathfrak{C}}}=c_{k}^{\mathfrak{C}}$. $(*)$ follows by Claim 1 .

Bueno et al. (2002, pp. 503-504) define partial homomorphisms between partial structures:

Definition 20 A partial homomorphism from partial structure $\tilde{\mathfrak{B}}$ to partial structure $\tilde{\mathfrak{C}}$ is a mapping $f: B \longrightarrow C$ for which the following holds: If $R_{i}^{\tilde{\mathfrak{B}},+} x_{1} \ldots x_{\lambda_{i}}$ then $R_{i}^{\tilde{\mathfrak{C}},+} f\left(x_{1}\right) \ldots f\left(x_{\lambda_{i}}\right)$ and if $R_{i}^{\tilde{\mathfrak{B}},-} x_{1} \ldots x_{\lambda_{i}}$ then $R_{i}^{\tilde{\mathfrak{C}},-} f\left(x_{1}\right) \ldots f\left(x_{\lambda_{i}}\right)$ for all $i \in I$, if $W_{\tilde{\mathfrak{B}}, j} x_{1} \ldots x_{\rho_{j}}$ then $W_{\tilde{\mathfrak{C}}, j} f\left(x_{1}\right) \ldots f\left(x_{\rho_{i}}\right)$ and for all $\left\langle x_{1}, \ldots, x_{\rho_{j}}\right\rangle \in W_{\tilde{\mathfrak{B}}, j}$, $f\left(F_{j}^{\tilde{\mathfrak{B}}} x_{1} \ldots x_{\rho_{j}}\right)=F_{j}^{\tilde{\mathfrak{C}}}\left(f\left(x_{1}\right) \ldots f\left(x_{\rho_{j}}\right)\right)$ for all $j \in J$, and $f\left(c_{k}^{\tilde{\mathfrak{A}}}\right)=c_{k}^{\tilde{\mathfrak{C}}}$ for all $k \in K$.

The claim that partial homomorphisms generalize standard homomorphisms (Bueno et al. 2002, p. 504) relies on an atypical definition of 'homomorphism', in which $R_{i}^{\mathfrak{B}} x_{1} \ldots x_{\lambda_{i}}$ if and only if $R_{i}^{\mathfrak{C}} f\left(x_{1}\right) \ldots f\left(x_{\lambda_{i}}\right)$ (Lutz 2015, p. 571), while the typical definition only requires that if $R_{i}^{\mathfrak{B}} x_{1} \ldots x_{\lambda_{i}}$, then $R_{i}^{\mathfrak{C}} f\left(x_{1}\right) \ldots f\left(x_{\lambda_{i}}\right)$ (Hodges 1993, p. 5). In the following, 'homomorphism' refers to the atypical notion as well.

Claim 7 Let $\mathbf{B}$ and $\mathbf{C}$ be the approximation sets corresponding to partial structures $\tilde{\mathfrak{B}}$ and $\tilde{\mathfrak{C}}$ with $\tilde{\Pi}=\varnothing$. Then $f$ is a partial homomorphism from $\tilde{\mathfrak{B}}$ to $\tilde{\mathfrak{C}}$ if and only if for every $\mathfrak{C} \in \mathbf{C}$ there is a $\mathfrak{B} \in \mathbf{B}$ such that $f$ is a homomorphism from $\mathfrak{B}$ to $\mathfrak{C}$.

Proof ' $\Rightarrow$ ': Let $f: B \longrightarrow C$ be a partial homomorphism from $\tilde{\mathfrak{B}}$ to $\tilde{\mathfrak{C}}$ and let $\mathfrak{C}$ be in C. By Claim $1, \mathfrak{C}$ is $\tilde{\mathfrak{C}}$-normal for $\varnothing$. By construction, $f$ is a homomorphism from $\mathfrak{B}$ to $\mathfrak{C}$ for any $\tilde{\mathfrak{B}}$-normal structure $\mathfrak{B}$ with $\operatorname{dom}(\mathfrak{B})=\operatorname{dom}(\tilde{\mathfrak{B}}), R_{i}^{\mathfrak{B}}=\stackrel{-1}{f}\left(R_{i}^{\mathfrak{C}}\right)$ for each

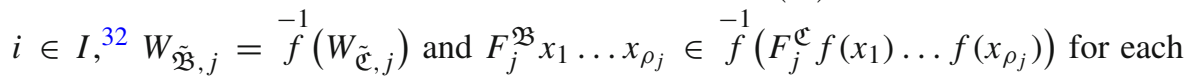
$\left\langle x_{1}, \ldots, x_{\rho_{j}}\right\rangle \in W_{\tilde{\mathfrak{B}}, j}, j \in J$, and $c_{k}^{\mathfrak{B}} \in \stackrel{-1}{f}^{-1}\left(c_{k}^{\mathfrak{C}}\right)$ for each $k \in K$. Such a structure exists because first, $R_{i}^{\tilde{\mathfrak{B}},+}=\stackrel{-1}{f}\left(R_{i}^{\tilde{\mathfrak{C}},+}\right) \subseteq \stackrel{-1}{f}\left(R_{i}^{\mathfrak{C}}\right)=R_{i}^{\mathfrak{B}}=B^{\lambda_{i}}-\stackrel{-1}{f}\left(B^{\lambda_{i}}-\right.$

32 ' $f$ ' $x$ ' denotes the pre-image of $x$ under function $f$, to be distinguished from the inverse function $f^{-1}$ applied to $x$. 
$\left.R_{i}^{\mathfrak{C}}\right) \subseteq B^{\lambda_{i}}-{ }^{-1}\left(R_{i}^{\tilde{\mathfrak{C}},-}\right)=B^{\lambda_{i}}-R_{i}^{\tilde{\mathfrak{B}},-}$. Second, $W_{\tilde{\mathfrak{B}}, j}={ }^{-1}\left(W_{\tilde{\mathfrak{C}}, j}\right)$ and hence $f\left(F_{j}^{\mathfrak{B}} x_{1} \ldots x_{\rho_{j}}\right)=F_{j}^{\mathfrak{C}} f\left(x_{1}\right) \ldots f\left(x_{\rho_{j}}\right)=F_{j}^{\tilde{\mathfrak{C}}} f\left(x_{1}\right) \ldots f\left(x_{\rho_{j}}\right)=f\left(F_{j}^{\tilde{\mathfrak{B}}} x_{1} \ldots x_{\rho_{j}}\right)$ for all $\left\langle x_{1}, \ldots, x_{\rho_{j}}\right\rangle \in W_{\tilde{\mathfrak{B}}}$. Third, $f\left(c_{k}^{\mathfrak{B}}\right)=c_{k}^{\mathfrak{C}}=c_{k}^{\tilde{\mathfrak{C}}}=f\left(c_{k}^{\tilde{\mathfrak{B}}}\right)$. Therefore $\mathfrak{B}$ can be chosen to be $\tilde{\mathfrak{B}}$-normal. By Claim $1, \mathfrak{B} \in \mathbf{B}$.

' $\Leftarrow$ ': Assume that $f: B \longrightarrow C$ is not a partial homomorphism from $\tilde{\mathfrak{B}}$ to $\tilde{\mathfrak{C}}$. Then there are an $i \in I$ and some $x_{1}, \ldots, x_{\lambda_{i}} \in B$ such that (i) $R_{i}^{\mathfrak{B},+} x_{1} \ldots x_{\lambda_{i}}$ and not $R_{i}^{\mathfrak{C},+} f\left(x_{1}\right) \ldots f\left(x_{\lambda_{i}}\right)$ or (ii) $R_{i}^{\mathfrak{B},-} x_{1} \ldots x_{\lambda_{i}}$ and not $R_{i}^{\mathfrak{C},-} f\left(x_{1}\right) \ldots f\left(x_{\lambda_{j}}\right)$, or there is a $j \in J$ such that for some $x_{1}, \ldots, x_{\rho_{j}} \in B$, (iii) $W_{\tilde{\mathfrak{B}}, j} x_{1} \ldots x_{\rho_{j}}$ and not $W_{\tilde{\mathfrak{C}}, j} f\left(x_{1}\right) \ldots f\left(x_{\rho_{j}}\right)$ or (iv) $W_{\tilde{\mathfrak{B}}, j} x_{1} \ldots x_{\rho_{j}}$ and $f\left(F_{j}^{\tilde{\mathfrak{B}}} x_{1} \ldots x_{\rho_{j}}\right) \neq$ $F_{j}^{\tilde{\mathfrak{C}}} f\left(x_{1}\right) \ldots f\left(x_{\rho_{j}}\right)$, or $(\mathrm{v})$ there is a $k \in K$ such that $f\left(c_{k}^{\tilde{\mathfrak{B}}}\right) \neq c_{k}^{\tilde{\mathcal{C}}}$. It is to be shown that $(*)$ there is a $\mathfrak{C} \in \mathbf{C}$ for which there is no $\mathfrak{B} \in \mathbf{B}$ such that $f$ is a homomorphism from $\mathfrak{B}$ to $\mathfrak{C}$.

If (i) holds for some $i \in I$ and $x_{1}, \ldots, x_{\lambda_{i}}$, then choose a $\tilde{\mathfrak{C}}$-normal structure in which $R_{i}^{\mathfrak{C}} f\left(x_{1}\right) \ldots f\left(x_{\lambda_{i}}\right)$ does not hold, and if (ii) holds for some $i \in I$ and $x_{1}, \ldots, x_{\lambda_{i}}$, then choose a $\tilde{\mathfrak{C}}^{\mathfrak{C}}$-normal structure with $R_{i}^{\mathfrak{C}} f\left(x_{1}\right) \ldots f\left(x_{\lambda_{i}}\right)$. Then, if $f$ is a homomorphism from $\mathfrak{B}$ to $\mathfrak{C}$, in case (i) $\mathfrak{B}$ is not $\tilde{\mathfrak{B}}$-normal because $R^{\mathfrak{B}} x_{1} \ldots x_{\lambda_{i}}$ does not hold even though $R^{\tilde{\mathfrak{B}},+} x_{1} \ldots x_{\lambda_{i}}$ does, and analogously for (ii). If (iii) or (iv) holds, choose $F_{j}^{\mathfrak{C}} f\left(x_{1}\right), \ldots, f\left(x_{\rho_{j}}\right) \neq f\left(F_{j}^{\tilde{\mathfrak{B}}} x_{1} \ldots x_{\rho_{j}}\right)$. Then, if $f$ is a homomorphism between $\mathfrak{B}$ and $\mathfrak{C}, \mathfrak{B}$ is not $\tilde{\mathfrak{B}}$-normal because $f\left(F_{j}^{\mathfrak{B}} x_{1} \ldots x_{\rho_{j}}\right) \neq f\left(F_{j}^{\tilde{\mathfrak{B}}} x_{1} \ldots x_{\rho_{j}}\right)$, and thus $F_{j}^{\mathfrak{B}} x_{1} \ldots x_{\rho_{j}} \neq F_{j}^{\tilde{\mathfrak{B}}} x_{1} \ldots x_{\rho_{j}}$. If (v) holds, $c_{k}^{\mathfrak{B}}=c_{k}^{\tilde{\mathfrak{B}}} \neq c_{k}^{\tilde{\mathfrak{C}}}=c_{k}^{\mathfrak{C}} .(*)$ follows by Claim 1 .

\section{Appendix B: Suárez's criticism of empirical adequacy}

According to a criticism by Suárez (1995, 2005), van Fraassen's notion of empirical adequacy does not, pace van Fraassen (1980, p. 12), capture what it means that a theory "saves the phenomena". The structure of Suárez's argument is simple: In the 1930s, electromagnetic theory was considered to save the phenomenon of superconductivity, but electromagnetic theory has no empirical substructure ${ }^{33}$ that is isomorphic to this phenomenon and is thus not empirically adequate. Therefore, the two notions cannot be equivalent.

In slightly more detail, Suárez $(2005, \S 6.1)$ considers the classic electromagnetic theory to include Maxwell's equations in matter, which give the relations between the electric field $\mathbf{E}$, magnetic field $\mathbf{H}$, the charge density $\rho$, the current density $\mathbf{j}$, the time $t$, and the speed of light $c$. It also includes the acceleration equation, which gives an additional relation between $\mathbf{H}$, the magnetic field $\mathbf{H}_{0}$ at the time a material transitions

\footnotetext{
33 With a reference to Suppes (2002, §62), Suárez (2005, p. 38) defines a substructure of $\mathfrak{T}$ to have possibly fewer relations than $\mathfrak{T}$. This is rather the definition of a relativized reduct, and misconstrues van Fraassen's notion of empirical adequacy and Suppes's definition. Suárez (2005, p. 39) weakens empirical adequacy even further (Lutz 2014b, §2.1), but since he argues that empirical adequacy is too strong, these changes do not threaten his conclusion.
} 
to its superconductive state (Suárez 2005, p. 53), the speed of light $c$, and the constant $\Lambda$; this equation was meant to deal with the behavior of the magnetic flux in superconductors. The acceleration equation therefore describes the empirical substructure of the classic electromagnetic theory as far as superconductivity is concerned. But the magnetic field $\mathbf{H}$ in superconductors in fact is, with slight idealizations, correctly described by the Londons' equation for $\Lambda, c$, and $\mathbf{H}$, which therefore describes the structure of the appearances.

There is, as is demanded for empirical adequacy, a bijection between the domains of the empirical substructure and the structure of the appearances, since, according to Suárez (2005, p. 54),

the two domains are isomorphic: for every physical entity (i.e. $\mathbf{j}, \mathbf{H}$, etc.) in the domain over which the 'acceleration equation' model is defined, there is a corresponding entity in the domain of the London model.

But the Londons' equation is incompatible with the acceleration equation for $\mathbf{H} \neq 0$, that is, their conjunction is inconsistent (Suárez 2005, p. 54), so that the structures cannot be isomorphic. There can also be no other empirical substructure of the classic electromagnetic theory that could be isomorphic to the Londons' equation when $\mathbf{H} \neq$ 0 , since then the theory would yield incompatible predictions for the same domain and would thus be inconsistent.

One could also take electromagnetic theory to consist only of Maxwell's equations, without the acceleration equation. This theory is compatible with the Londons' equation even when $\mathbf{H} \neq 0$, but, says Suárez (2005, p. 55), it

can provide neither an accurate nor an inaccurate representation of superconductivity phenomena-it can provide no representation at all! The phenomenon of superconductivity simply lies outside the theory's domain of empirical adequacy, and the question of embedding [...] of [the phenomena] simply does not arise.

Therefore Suárez concludes that neither classical electromagnetic theory nor electromagnetic theory according to Maxwell's equations is empirically adequate in light of the appearances of superconductivity.

There are a number of problems with Suárez's argument. The main one is its overly impressionistic nature. Suárez does not define a family of structures for the classic electromagnetic theory, nor does he define its empirical substructures. Instead, he mentions the equations that hold for the functions that occur in the respective structures. For this reason, his description of the domain of the theory may seem more plausible than it is: According to Suárez, the domain of the empirical substructure contains the physical quantities $\mathbf{j}$ and $\mathbf{H}$, so that, apparently, the domain of the classical electromagnetic theory contains the physical quantities that occur in Maxwell's equations and the acceleration equation: $\mathbf{E}, \mathbf{H}, \mathbf{H}_{0}, \Lambda, \mathbf{j}, c$, and $\rho$. (I am unsure about the way in which time development would be represented in this formalization.) The relations that hold between these objects are thus, presumably, the equations themselves. Suárez (2005, p. 54) furthermore seems to consider any equation that follows from these equations to be part of the structure, since he claims on the ground that one equation follows 
from both the Londons' and the acceleration equation that "at least one relation over the domain is isomorphic".

The empirical substructures of the classic electromagnetic theory therefore are structures that contain some, but not necessarily all, of the quantities that occur in the classic electromagnetic theory itself. The first part of Suárez's argument then is that the relations that are given by Maxwell's equation and the acceleration equation cannot be fulfilled by the same objects that fulfill the relation given by the Londons' equation, since the equations are incompatible. The second, rather loosely formulated part of his argument could be reconstructed as follows: Maxwell's equations alone do not entail the Londons' equation, and therefore there is no relation in any structure of the electromagnetic theory (as described by Maxwell's equations) that corresponds to the Londons' equation. Since any substructure of a structure has the same relations as the structure itself, no empirical substructure of the electromagnetic theory can be isomorphic to a structure that contains the Londons' equation. ${ }^{34}$

Suárez's formalization of the electromagnetic theory is very different from a formalization in van Fraassen's spirit, which would rather describe the state space of the systems with the respective physical quantities (van Fraassen 1970, §§3-5). In the state space description of a classical system of finitely many objects, each fundamental quantity of each object is assigned a dimension of the state space, so that at each time, the values of all quantities of all objects are given by a vector in state space. Laws are expressed as restrictions on state space. For a classical field, each point in physical space can have a different value, so that each quantity of each point in physical space would have to be assigned a dimension in state space. This would result in a non-denumerably dimensional state space. ${ }^{35}$

Not as close to van Fraassen's preferred formalization but still typical in the semantic view (van Fraassen 1980, p. 67) would be to give a structure with physical spacetime in its domain, the physical quantities $\mathbf{E}, \mathbf{H}$, and $\mathbf{j}$ as functions from spacetime points to triples of real numbers, $\mathbf{H}_{0}$ as a function from space points to triples of real numbers, the physical quantity $\rho$ as a function from spacetime points to real numbers, and $c$ and $\Lambda$ as real constants. Maxwell's, the Londons', and the acceleration equation would then be demanded to hold between these functions and constants of the structures. ${ }^{36}$ Since the Londons' equation describes superconductors, each of its models (and specifically each of its models for $\mathbf{H} \neq 0$ ) must be isomorphic to some empirical substructure of electromagnetic theory. Otherwise, electromagnetic theory is not empirically adequate. In a formalization that follows this sketch, Suárez's argument against the empirical adequacy of classic electromagnetic theory is valid, since the functions and constants cannot simultaneously fulfill two incompatible equations. However, Suárez's argument against the empirical adequacy of the electromagnetic theory as described by Maxwell's equations alone fails: A structure with the points in physical space and the real numbers as its domain, and the physical quantities

\footnotetext{
34 This also holds for Suárez's use of relativized reducts instead of substructures, since the relativized reduct of a structure $\mathfrak{B}$ cannot contain any relations not contained in $\mathfrak{B}$.

35 It is unfortunately not clear how the notion of empirical adequacy, which is defined for theories described in terms of model theory, is to be translated into van Fraassen's earlier notion of a state space (Lutz 2017, p. 328).

36 This is essentially the formalization chosen by Suárez and Cartwright (2008, p. 74).
} 
given above as its relations is a structure of the electromagnetic theory if and only if the physical quantities fulfill Maxwell's equations. Since Maxwell's equations are compatible with the Londons' equations for restricted domains, there are empirical substructures of the electromagnetic theory that are isomorphic to the structure of the phenomenon of superconductivity as described by the Londons' equation. Thus the electromagnetic theory as described by Maxwell's equations is empirically adequate for the appearances of superconductivity. ${ }^{37}$

Therefore, Suárez's criticism is not fatal for the concept of empirical adequacy, because in a plausible formalization, only the classic electromagnetic theory fails to be empirically adequate. And this is as it should be, since the acceleration equation describes the appearances incorrectly, and the conjunction of Maxwell's equation and the acceleration equation can therefore not be considered to save the appearances.

\section{Appendix C: Bueno's reply to Suárez}

\section{Appendix C.1: Partial empirical adequacy}

According to Bueno (1997, §4.4, my notation), classic electromagnetic theory as described by Maxwell's equation and the acceleration equation is partially empirically adequate, so that his generalization of empirical adequacy to partial empirical adequacy avoids Suárez first criticism:

Indeed, the kind of new relation discovered at the phenomenological level might be represented by one of those partial relations that initially were not defined for some elements of the domain under investigation (whose elements thus belong to its $R^{\circ}$-component), and with the introduction of further bits of information, came to be defined - hence, the relevant elements belong then either to its domain ( $R^{+}$-component), or to its complement ( $R^{-}$-component).

This reply to Suárez is rather vague, but it seems that the Londons' equation is the "new relation discovered" between objects of the domain, or better: It is the discovery that some relation holds between objects of the domain (Bueno is thus relying on Suárez's formalization).

A major problem with this reply is that it is not clear in what way the notion of partial empirical adequacy is time dependent, and thus it is not clear how the discovery that some elements of the domain have some relation can be represented as partial empirical adequacy. Another problem is that it is utterly artificial to use partial relations to formalize a situation in which an equation is discovered to hold between physical quantities: Before the Londons' equation was discovered to hold for $\Lambda, c$, and $\mathbf{H}$, no one was of the opinion that it held (or did not hold) for, say $c, c$, and $\mathbf{E}$ and was wondering whether it also held for other physical quantities of the above list. Staying in Suárez's formalization, it was rather that the Londons discovered a new relation-just as Bueno puts it informally in his reply quoted above. Finally, Suárez $(2005, \S 6.2)$ points out

37 This result is obvious when considering that syntactically, a theory is empirically adequate given the appearances if and only if the theory's description is compatible with the description of the appearances (Lutz 2014a, claim 1). 
that, since the acceleration equation is incompatible with the Londons' equation, $\Lambda$, $c$, and $\mathbf{H}$ are, according to the acceleration equation, certainly not in the relation described by the Londons' equation. That is, in any partial appearance $\tilde{\mathfrak{A}}, R_{i}^{\tilde{\mathfrak{A}}}+{ } \Lambda c \mathbf{H}$ holds, ${ }^{38}$ where $R_{i}$ is given by the Londons' equation. The partial isomorphism $f$ to the empirical substructure then gives $R_{i}^{\tilde{\mathfrak{E}},+} f(\Lambda) f(c) f(\mathbf{H})$ for the analogue of the Londons' equation in the theory. But this would lead to inconsistency, since according to the classic electromagnetic theory, $R_{i}^{\tilde{\mathfrak{E}},-} f(\Lambda) f(c) f(\mathbf{H})$.

In the formalization that I have sketched, the classic electromagnetic theory is not partially empirically adequate either: The domain of the Londons' equation consists of superconductors, and its terms are $\Lambda, c$, and $\mathbf{H}$. In each appearance of superconductivity, these terms' interpretations fulfill the Londons' equation. Since this is known, every partial structure $\tilde{\mathfrak{A}}$ of the appearances must be such that every $\tilde{\mathfrak{A}}$-normal structure fulfills the Londons' equation. The classic electromagnetic theory, on the other hand, must be such that for no partial empirical substructure $\tilde{\mathfrak{E}}$, there is an $\tilde{\mathfrak{E}}$-normal structure that fulfills the Londons' equation, since it is part of the theory that the Londons' equation is not fulfilled by superconductors. Since no $\tilde{\mathfrak{A}}$-normal and $\tilde{\mathfrak{E}}$-normal structures are isomorphic, $\tilde{\mathfrak{A}}$ and $\tilde{\mathfrak{E}}$ are not partially isomorphic either, by Claims 1 and 6 ("Appendix A"). Electromagnetic theory as described by Maxwell's equations is partially empirically adequate, however, because partial empirical adequacy generalizes empirical adequacy and electromagnetic theory is empirically adequate.

\section{Appendix C.2: Pragmatic empirical adequacy}

The requirements for the pragmatic empirical adequacy of a theory are almost trivially weak. This has the intended effect of avoiding Suárez's counterexample if the relation described by the Londons' equation is considered to determine the structure of the data (the lowest level of the models of the phenomena), not the appearance at the highest level: While the relation between $\Lambda, c$, and $\mathbf{H}$ described by the Londons' equation has to hold for the structure of the data, the structure of the appearances can contain any relation between $\Lambda, c$, and $\mathbf{H}$ under one further condition: At least one element in the domain of the highest level must have been measured. Apart from this condition, one can choose any structure with the acceleration equation as relation between $\Lambda$, $c$, and $\mathbf{H}$. Therefore the classic electromagnetic theory is pragmatically empirically adequate.

In the formalization of the classic electromagnetic theory that I have sketched, the case is similar: The domain of the Londons' equation contains superconductors, and its terms are $\Lambda, c$, and $\mathbf{H}$. (To apply Bueno's definitions for relational structures, the theory first has to be reformulated to contain only relations rather than functions and constants.) Since the domain of the appearances, the volume of the superconductors over time, has infinite cardinality, there is no restriction on the domain of the highest level of the models of the phenomena. One can now choose a structure with the same terms and at least one measured object in its domain in which the classic electromagnetic theory is true, so that the theory is pragmatically empirically adequate. Of

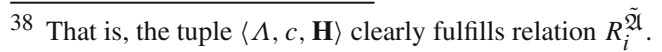


course, this strength of Bueno's definition is also a huge weakness, since any other theory with the same relation symbols is pragmatically empirically adequate as well.

In summary, the notion of partial empirical adequacy does not avoid Suárez's criticism, and the notion of pragmatic empirical adequacy does avoid it, but only on pain of (almost) triviality. Neither failure poses a problem for empirical adequacy or the project of constructive empiricism in general, however, because Suárez's criticism is an artifact of an ill-chosen formalization of the theory of electromagnetism.

\section{References}

Annett, J. F. (2004). Superconductivity, superfluids, and condensates (Vol. 5). Oxford: Oxford University Press.

Bogen, J., \& Woodward, J. (1988). Saving the phenomena. The Philosophical Review, 97(3), 303-352. https://doi.org/10.2307/2185445.

Bueno, O. (1997). Empirical adequacy: A partial structures approach. Studies in the History and Philosophy of Science, 28(4), 585-610. https://doi.org/10.1016/S0039-3681(97)00012-5.

Bueno, O. (1999). What is structural empiricism? Scientific change in an empiricist setting. Erkenntnis, 50, 59-85. https://doi.org/10.1023/A:1005434915055.

Bueno, O. (2000). Empiricism, scientific change and mathematical change. Studies in the History and Philosophy of Science, 31(2), 269-296. https://doi.org/10.1016/S0039-3681(99)00037-0.

Bueno, O., \& Colyvan, M. (2011). An inferential conception of the application of mathematics. Noûs, 45(2), 345-374. https://doi.org/10.1111/j.1468-0068.2010.00772.x.

Bueno, O., \& de Souza, E. G. (1996). The concept of quasi-truth. Logique \& Analyse, 153-154, 183-199.

Bueno, O., French, S., \& Ladyman, J. (2002). On representing the relationship between the mathematical and the empirical. Philosophy of Science, 69(3), 497-518. https://doi.org/10.1086/342456.

Chang, C. C., \& Keisler, H. J. (1990). Model theory (3rd ed., Vol. 73). Amsterdam: Elsevier. 3rd impression.

da Costa, N. C. A., Bueno, O., \& French, S. (1998). The logic of pragmatic truth. Journal of Philosophical Logic, 27(6), 603-620. https://doi.org/10.1023/A:1004304228785.

da Costa, N. C. A., \& French, S. (1990). The model-theoretic approach in the philosophy of science. Philosophy of Science, 57(2), 248-65. https://doi.org/10.1086/289546.

da Costa, N. C. A., \& French, S. (2000). Models, theories, and structures: Thirty years on. Philosophy of Science, 67, S116-S127. https://doi.org/10.1086/392813.

da Costa, N. C. A., \& French, S. (2003). Science and partial truth: A unitary approach to models and scientific reasoning. Oxford: Oxford University Press. https://doi.org/10.1093/019515651x.001.0001.

Ebbinghaus, H.-D., Flum, J., \& Thomas, W. (1994). Mathematical logic. New York, NY: Springer. https:// doi.org/10.1007/978-1-4757-2355-7.

Eklund, M. (2009). Carnap and ontological pluralism. In D. Chalmers, D. Manley, \& R. Wasserman (Eds.), Metametaphysics: New essays on the foundations of ontology (pp. 130-156). Oxford: Oxford University Press.

Fine, K. (1997). Vagueness, truth and logic. In R. Keefe \& P. Smith (Eds.), Vagueness. A reader (pp. 119-150). Cambridge, MA: The MIT Press.

French, S. (2000). The reasonable effectiveness of mathematics: Partial structures and the application of group theory to physics. Synthese, 125, 103-120. https://doi.org/10.1023/A:1005246608001.

French, S. (2003). A model-theoretic account of representation (or, I don't know much about art... but I know it involves isomorphism). Philosophy of Science, 70, 1472-1483. https://doi.org/10.1086/377423.

French, S. (2014). The structure of the world. Oxford: Oxford University Press. https://doi.org/10.1093/ acprof:oso/9780199684847.001.0001.

French, S., \& Ladyman, J. (1997). Superconductivity and structures: Revisiting the London account. Studies in History and Philosophy of Science Part B: Studies In History and Philosophy of Modern Physics, 28(3), 363-393. https://doi.org/10.1016/S1355-2198(97)00013-0.

French, S., \& Ladyman, J. (1999). Reinflating the semantic approach. International Studies in the Philosophy of Science, 13(2), 103-121. https://doi.org/10.1080/02698599908573612.

Hammett, D. (1928). This king business. Mystery Stories. (References are to the edited reprint (Hammett, 1989, 115-170)) 
Hammett, D. (1989). The big knockover. New York, NY: Random House. (Edited and with an introduction by Lillian Hellman)

Hodges, W. (1993). Model theory (Vol. 42). Cambridge: Cambridge University Press. https://doi.org/10. 1017/CBO9780511551574.

Lutz, S. (2014a). Empirical adequacy in the Received View. Philosophy of Science, 81(5), 1171-1183. https://doi.org/10.1086/677886.

Lutz, S. (2014b). Generalizing empirical adequacy I: Multiplicity and approximation. Synthese, 191, 31953225. https://doi.org/10.1007/s11229-014-0440-3.

Lutz, S. (2015). Partial model theory as model theory. Ergo, 2(22), 563-580. https://doi.org/10.3998/ergo. 12405314.0002.022.

Lutz, S. (2017). What was the syntax-semantics debate in the philosophy of science about? Philosophy and Phenomenological Research, 95(2), 319. https://doi.org/10.1111/phpr.12221.

Mikenberg, I., da Costa, N. C. A., \& Chuaqui, R. (1986). Pragmatic truth and approximation to truth. The Journal of Symbolic Logic, 51(1), 201-221. https://doi.org/10.2307/2273956.

Monton, B., \& Mohler, C. (2008). Constructive empiricism. In E. N. Zalta (Ed.), The Stanford encyclopedia of philosophy (Winter 2008 ed.). Stanford: The Metaphysics Research Lab, Center for the Study of Language and Information, Stanford University. http://plato.stanford.edu/archives/win2008/entries/ constructive-empiricism/.

Przełęcki, M. (1976). Fuzziness as multiplicity. Erkenntnis, 10, 371-380. https://doi.org/10.1007/ BF00214731.

Suárez, M. (1995). How theories save phenomena: A case against embedding. (Unpublished typescript)

Suárez, M. (2005). The semantic view, empirical adequacy, and application. Crítica, Revista Hispanoamericana de Filosofia, 37(109), 29-63.

Suárez, M., \& Cartwright, N. (2008). Theories: Tools versus models. Studies in History and Philosophy of Modern Physics, 39, 62-81. https://doi.org/10.1016/j.shpsb.2007.05.004.

Suppes, P. (1962). Models of data. In E. Nagel, P. Suppes, \& A. Tarski (Eds.), Logic, methodology, and philosophy of science: Proceedings of the 1960 international congress (pp. 252-261). Stanford: Stanford University Press.

Suppes, P. (2002). Representation and invariance of scientific structures. Stanford, CA: CSLI Publications. van Fraassen, B. C. (1970). On the extension of Beth's semantics of physical theories. Philosophy of Science, 37(3), 325-339. https://doi.org/10.1086/288311.

van Fraassen, B. C. (1980). The scientific image. Oxford: Clarendon Press. https://doi.org/10.1093/ 0198244274.001 .0001 .

van Fraassen, B. C. (2008). Scientific representation: Paradoxes of perspective. Oxford: Clarendon Press. https://doi.org/10.1093/acprof:oso/9780199278220.001.0001.

Publisher's Note Springer Nature remains neutral with regard to jurisdictional claims in published maps and institutional affiliations. 